\title{
Heart Rate Variability in the Perinatal Period: A Critical and Conceptual Review
}

\begin{abstract}
Marco Chiera ${ }^{1,2 t}$, Francesco Cerritelli ${ }^{\star \star t}$, Alessandro Casini ${ }^{1}$, Nicola Barsotti ${ }^{1,2}$, Dario Boschiero ${ }^{3}$, Francesco Cavigioli ${ }^{4}$, Carla G. Corti ${ }^{5}$ and Andrea Manzotti $1,4,6$

${ }^{1}$ Research and Assistance for Infants to Support Experience Lab, Foundation Center for Osteopathic Medicine Collaboration, Pescara, Italy, ${ }^{2}$ Research Commission on Manual Therapies and Mind-Body Disciplines, Società Italiana di Psico Neuro Endocrino Immunologia, Rome, Italy, ${ }^{3}$ BioTekna - Biomedical Technologies, Venice, Italy, ${ }^{4}$ Neonatal Intensive Care Unit, "V. Buzzi" Children's Hospital, Azienda Socio Sanitaria Territoriale Fatebenefratelli-Sacco, Milan, Italy, ${ }^{5}$ Pediatric Cardiology Unit-Pediatric Department, Azienda Socio Sanitaria Territoriale Fatebenefratelli-Sacco, Milan, Italy, ${ }^{6}$ Research Department, SOMA, Istituto Osteopatia Milano, Milan, Italy
\end{abstract}

Neonatal intensive care units (NICUs) greatly expand the use of technology. There is a need to accurately diagnose discomfort, pain, and complications, such as sepsis, mainly before they occur. While specific treatments are possible, they are often timeconsuming, invasive, or painful, with detrimental effects for the development of the infant. In the last 40 years, heart rate variability (HRV) has emerged as a noninvasive measurement to monitor newborns and infants, but it still is underused. Hence, the present paper aims to review the utility of HRV in neonatology and the instruments available to assess it, showing how HRV could be an innovative tool in the years to come. When continuously monitored, HRV could help assess the baby's overall wellbeing and neurological development to detect stress-/painrelated behaviors or pathological conditions, such as respiratory distress syndrome and hyperbilirubinemia, to address when to perform procedures to reduce the baby's stress/pain and interventions, such as therapeutic hypothermia, and to avoid severe complications, such as sepsis and necrotizing enterocolitis, thus reducing mortality. Based on literature and previous experiences, the first step to efficiently introduce HRV in the NICUs could consist in a monitoring system that uses photoplethysmography, which is low-cost and non-invasive, and displays one or a few metrics with good clinical utility. However, to fully harness HRV clinical potential and to greatly improve neonatal care, the monitoring systems will have to rely on modern bioinformatics (machine learning and artificial intelligence algorithms), which could easily integrate infant's HRV metrics, vital signs, and especially past history, thus elaborating models capable to efficiently monitor and predict the infant's clinical conditions. For this reason, hospitals and institutions will have to establish tight collaborations between the obstetric, neonatal, and pediatric departments: this way, healthcare would truly improve in every stage of the perinatal period (from conception to the first years of life), since information about patients' health would flow freely among different professionals, and high-quality research could be performed integrating the data recorded in those departments.

Keywords: autonomic nervous system, vagus, newborns, preterm infants, neonatology, NICU, photoplethysmography, HRV 


\section{INTRODUCTION}

The neonatology field is growing in complexity (Biban, 2010). In essence, newborns can show many comorbidities associated with prematurity (WHO, 2016), labor complications (Tribe et al., 2018), and maternal and perinatal stress (Frasch et al., 2007; Babenko et al., 2015; Lobmaier et al., 2020), whereas the neonatal intensive care units (NICUs) are increasing the use of technology to better take care of fetuses, newborns, and infants (Biban, 2010; Chock et al., 2015).

However, many obstacles need to be overcome to efficiently assess and manage infants' conditions: distress, pain, and sepsis need valid and reliable gauges to detect them before they happen (Cremillieux et al., 2018; Rashwan et al., 2019), but several procedures may be time-consuming (Jeng et al., 2000; Als et al., 2005; Cremillieux et al., 2018), invasive, and painful with short- and long-term negative consequences (Holsti et al., 2006; Pillai Riddell et al., 2015).

In the last 40 years, heart rate variability (HRV) has emerged as a reliable and non-invasive measure to monitor preterm and term newborns (Task Force of the European Society of Cardiology the North American Society of Pacing Electrophysiology, 1996). HRV evaluates the heart rate (HR) fluctuation-the variability of the time intervals between successive heartbeats-, and during the years, more and more techniques have appeared to improve its analysis, from which several metrics can be extracted (Table 1; Bravi et al., 2011; Thiriez et al., 2015; Javorka et al., 2017; Oliveira et al., 2019b; Patural et al., 2019).

Several studies showed that HRV correlates with the newborn's stress and stress-related behaviors (Gardner et al., 2018; Hashiguchi et al., 2020), and that it could predict the baby's overall wellbeing and future neurological development. HRV could also accurately identify short- and long-term complications, such as the risk of sepsis (Javorka et al., 2017; Oliveira et al., 2019b; Kumar et al., 2020). HRV was also able to reveal the impact of prenatal stress on fetal brain development (Frasch et al., 2007; Lobmaier et al., 2020).

Despite these results, HRV is still underused in NICUs. Although in the 1960s, one of the first evidences published was about the alteration of HRV metrics preceding fetal distress (Task Force of the European Society of Cardiology the North

\footnotetext{
Abbreviations: AI, artificial intelligence; AMP, amplitude fluctuations; ANS, autonomic nervous system; AS, active sleep; BSG, ballistography; CAN, central autonomic network; CAP, cholinergic anti-inflammatory pathway; CIMVA, continuous individual multiorgan variability analysis; CNS, central nervous system; COMP, complexity; CPAP, continuous positive airway pressure; CTG, cardiotocography; ECG, electrocardiogram; fABAS, fetal autonomic brain age scale; fECG, fetal electrocardiogram; fHRV, fetal heart rate variability; fMCG, fetal magnetocardiography; FSE, fetal scalp electrode; GA, gestational age; HeRO, Heart Rate Observation system; HIE, hypoxic-ischemic encephalopathy; HR, heart rate; HRC, heart rate characteristics; HRV, heart rate variability; IUGR, intrauterine growth restriction; ML, machine learning; NEC, necrotizing enterocolitis; NIPE, newborn infant parasympathetic evaluation; PNS, parasympathetic nervous system; PPG, photoplethysmography; QS, quiet sleep; PNS, parasympathetic nervous system; PRSA, phase rectified signal averaging; PRV, pulse rate variability; RSA, respiratory sinus arrhythmia; SIDS, sudden infant death syndrome; SNS, sympathetic nervous system; $\mathrm{SpO}_{2}$, partial oxygen saturation; vPPG, non-contact video-photoplethysmography.
}

American Society of Pacing Electrophysiology, 1996); to date, some authors argued that there is a lack of understanding of the meaning of HRV metrics in infants: in fact, HRV has been studied especially in adults, and the autonomic nervous system (ANS) behaves differently in newborns, especially in preterm infants (Joshi et al., 2019b). Notwithstanding these contradictions, the only successful example of integration of HRV in NICUs is the Heart Rate Observation (HeRO) monitor developed by J. Randall Moorman's team. The HeRO analyzes bedside electrocardiogram (ECG) in real-time and integrates various HRV metrics to calculate the "HRC index," which can predict the risk of sepsis within $24 \mathrm{~h}$ in both preterms and very low birth weight infants (Andersen et al., 2019; Kumar et al., 2020).

However, based on the available literature and on the potential research to be developed, there is a need to further explore the use of HRV in neonatology.

HRV may provide such useful insights since it correlates with the ANS development and functioning. The ANS regulates organic development and connects with the organism's ability to cope with stressors, as well as with cognitive and emotional development (Thayer et al., 2012; Jennings et al., 2015; Schneider et al., 2018; Oliveira et al., 2019b).

To be an innovative tool useful for neonatologists, HRV measurement should rely on a technology that gives reliable metrics with a clear clinical meaning. Harnessing the positive experiences, such as the use of the HeRO monitor, it is paramount to create a system that continuously records HRV and expresses scores that could correlate with the baby's clinical condition and help monitor its evolution (Zhao et al., 2016; Hayano and Yuda, 2019; Pernice et al., 2019b; Kumar et al., 2020).

For this purpose, modern machine learning (ML) and artificial intelligence (AI) algorithms could play a crucial role: through their computational power, they could define models capable of managing the complex physiological interactions between HRV, ANS, and the whole organism, thus boosting our ability to predict the infant's prognosis. Indeed, we already have experiences about the clinical usefulness of ML in both neonatology (Semenova et al., 2018; Ostojic et al., 2020) and HRV analysis (Chiew et al., 2019; Lin et al., 2020).

ML/AI algorithms could also integrate clinical data of different hospital departments, i.e., obstetric, neonatal, and pediatric. Indeed, free clinical data and medical devices sharing among the departments involved in the perinatal care (from conception to the first years of life) would allow clinicians to better understand the prenatal and developmental factors underlying adverse neonatal outcomes (e.g., brain injury) and to better treat them.

Therefore, the present paper aims to address the HRV usefulness in neonatology to prospect it as an innovative tool in the years to come. This focused review is divided into three sections: (1) the first section describes briefly the HRV metrics and examines the relationship between ANS and HRV in fetuses and newborns; (2) the second section examines the technology available in the NICU, how to monitor HRV efficiently, and the usefulness of real-time HRV; and (3) the third and final section will summarize the main findings and outline future perspectives for the clinical use of real-time HRV in the neonatal field, with a brief subsection about its usefulness in low-income countries. 


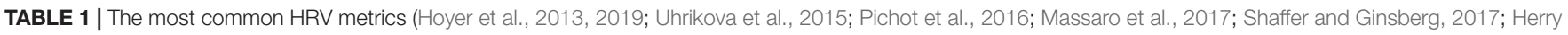
et al., 2019; Oliveira et al., 2019b; Patural et al., 2019; Frasch et al., 2020).

\begin{tabular}{|c|c|c|}
\hline Metric & Unit & Definition \\
\hline HR & bpm & HR (number of heart beats per minute) \\
\hline \multicolumn{3}{|l|}{ Time-domain } \\
\hline SDNN & ms & Standard deviation of NN intervals \\
\hline SDRR & ms & Standard deviation of RR intervals \\
\hline SDANN & ms & Standard deviation of the average NN intervals for each 5 min segment of a $24 \mathrm{~h} H R V$ recording \\
\hline SDNN Index & ms & Mean of the standard deviations of all the NN intervals for each 5 min segment of a $24 \mathrm{~h} H \mathrm{HR}$ recording \\
\hline NNx & & Number of adjacent NN intervals that differ from each other by more than x ms (e.g., 5, 20, 25, and 50 ms) \\
\hline pNNx & $\%$ & Percentage of successive NN intervals that differ by more than x ms (e.g., 5, 20, 25, and 50 ms) \\
\hline RMSSD & ms & Root mean square of consecutive RR interval differences \\
\hline SDSD & $\mathrm{ms}$ & Standard deviation of consecutive RR differences \\
\hline \multicolumn{3}{|l|}{ Frequency-domain } \\
\hline ULF Power & $m s^{2}$ & Absolute power of the ultra-low-frequency band $(\leq 0.003 \mathrm{~Hz})$ \\
\hline VLF Power & $m s^{2}$ & Absolute power of the very-low-frequency band $(0.0033-0.04 \mathrm{~Hz})$ \\
\hline LF Peak & $\mathrm{Hz}$ & Peak frequency of the low-frequency band $(0.04-0.2 \mathrm{~Hz}$ for newborns and $0.04-0.15 \mathrm{~Hz}$ for infants) \\
\hline \multirow[t]{3}{*}{ LF Power } & $m s^{2}$ & Absolute power of the low-frequency band $(0.04-0.2 \mathrm{~Hz}$ for newborns and $0.04-0.15 \mathrm{~Hz}$ for infants) \\
\hline & $\mathrm{Nu}$ & Relative power of the low-frequency band $(0.04-0.2 \mathrm{~Hz}$ for newborns and $0.04-0.15 \mathrm{~Hz}$ for infants) in normal units \\
\hline & $\%$ & Relative power of the low-frequency band $(0.04-0.2 \mathrm{~Hz}$ for newborns and $0.04-0.15 \mathrm{~Hz}$ for infants) \\
\hline HF Peak & $\mathrm{Hz}$ & Peak frequency of the high-frequency band $(0.20-2.00 \mathrm{~Hz}$ for newborns and $0.20-1.40 \mathrm{~Hz}$ for infants) \\
\hline \multirow[t]{3}{*}{ HF Power } & $m s^{2}$ & Absolute power of the high-frequency band $(0.20-2.00 \mathrm{~Hz}$ for newborns and $0.20-1.40 \mathrm{~Hz}$ for infants) \\
\hline & $\mathrm{Nu}$ & Relative power of the high-frequency band $(0.20-2.00 \mathrm{~Hz}$ for newborns and $0.20-1.40 \mathrm{~Hz}$ for infants) in normal units \\
\hline & $\%$ & Relative power of the high-frequency band $(0.20-2.00 \mathrm{~Hz}$ for newborns and $0.20-1.40 \mathrm{~Hz}$ for infants) \\
\hline LF/HF & $\%$ & Ratio of LF-to-HF power \\
\hline VLF/LF & $\%$ & Ratio between very-low $(0.02-0.08 \mathrm{~Hz})$ and low $(0.08-0.2 \mathrm{~Hz})$ frequency band power \\
\hline LFn & & $\begin{array}{l}\text { Normalized power in the low-frequency band of the ECG spectrogram }(0.04-0.2 \mathrm{~Hz} \text { for newborns and } 0.04-0.15 \mathrm{~Hz} \text { for } \\
\text { infants), i.e., low-frequency power in relation to total power }\end{array}$ \\
\hline HFn & & $\begin{array}{l}\text { Normalized power in the high-frequency band of the ECG spectrogram }(0.20-2.00 \mathrm{~Hz} \text { for newborns and } 0.04-0.15 \mathrm{~Hz} \text { for } \\
\text { infants), i.e., high-frequency power in relation to total power }\end{array}$ \\
\hline Total power (TP) & $m s^{2}$ & Total power of the ECG spectrogram \\
\hline \multicolumn{3}{|l|}{ Non-linear } \\
\hline S & $\mathrm{ms}$ & Area of the ellipse that represents total HRV \\
\hline SD1 & ms & Poincaré plot standard deviation perpendicular the line of identity \\
\hline SD2 & ms & Poincaré plot standard deviation along the line of identity \\
\hline $\mathrm{CSI}$ & $\%$ & Cardiac Sympathetic Index-SD1/SD2 \\
\hline $\mathrm{CVI}$ & & Cardiac Vagal Index-log (SD1*SD2) \\
\hline SVT & & Short-term variability of consecutive beat-to-beat data obtained through Poincaré analysis \\
\hline LTV & & Long-term variation of consecutive beat-to-beat data obtained through Poincaré analysis \\
\hline HRV Triangular Index & & Integral of the density of the RR interval histogram divided by its height \\
\hline HRV Index & & Number of all RR intervals divided by the number of RR intervals at the highest point of the RR histogram \\
\hline TINN & ms & $\begin{array}{l}\text { Triangular Interpolation of the NN Interval Histogram - the length of the basis of the minimum square difference of the triangular } \\
\text { interpolation for the highest value of the RR histogram or the normalized width of the base of the RR histogram }\end{array}$ \\
\hline Parseval Index & & Ratio between the square root of the sum of LF and HF powers and the value of SDNN \\
\hline$\%$ DET & & $\begin{array}{l}\text { Percentage of determinism of a time series from recurrence quantification analysis }(R Q A) \text { : it detects the predictability of } \\
\text { dynamical systems }\end{array}$ \\
\hline ShanEn & & Shannon Entropy-uncertainty of a random variable \\
\hline ApEn & & Approximate entropy, which measures the regularity and complexity of a time series \\
\hline SampEn & & Sample entropy, which measures the regularity and complexity of a time series \\
\hline MSEX & & Multiscale entropy at coarse graining level $x$ \\
\hline $\operatorname{gMSE}(x)$ & bit norm & Generalized multiscale entropy at coarse graining level x of NN interval series \\
\hline QSE & & Quadratic Sample Entropy \\
\hline KLPE & & Kullback-Leibler permutation entropy \\
\hline $\mathrm{AC}$ & & Acceleration capacity (detection of sequences of two successive RR beats that decrease) obtained through PRSA \\
\hline DC & & Deceleration capacity (detection of sequences of two successive RR beats that increase) obtained through PRSA \\
\hline
\end{tabular}


TABLE 1 | Continued

\begin{tabular}{|c|c|c|}
\hline Metric & Unit & Definition \\
\hline ACstx & & Acceleration capacity, slope, and step value at coarse graining level x \\
\hline DFA $\alpha 1$ & & Detrended fluctuation analysis, which describes short-term fluctuations \\
\hline DFA $\alpha 2$ & & Detrended fluctuation analysis, which describes long-term fluctuations \\
\hline DFA $\alpha_{S}$ & & Detrended fluctuation analysis, which describes short-term fluctuations \\
\hline DFA $\alpha_{L}$ & & Detrended fluctuation analysis, which describes long-term fluctuations \\
\hline $\mathrm{RMS}_{S}$ & & Root mean square from detrended fluctuation analysis, which describes short-term fluctuations \\
\hline $\mathrm{RMS}_{\mathrm{L}}$ & & Root mean square from detrended fluctuation analysis, which describes long-term fluctuations \\
\hline SDLE $\alpha$ & & Scale-dependent Lyapunov exponent slope \\
\hline Skewness & a.u. & Skewness of NN interval series \\
\hline Asyml & & $\begin{array}{l}\text { Multiscale time irreversibility asymmetry index: it is the degree of temporal asymmetry and lack of invariance of the statistical } \\
\text { properties of a signal }\end{array}$ \\
\hline $\mathrm{D}_{2}$ & & Correlation dimension, which estimates the minimum number of variables required to construct a model of system dynamics \\
\hline
\end{tabular}

\section{THE ANS PHYSIOLOGY UNDERLYING HRV AND ITS USE IN THE NEONATAL FIELD}

\section{A Brief Introduction on HRV Metrics}

From the work of the Task Force for HRV analysis in 1996, which constitutes the foundation for the majority of the papers on HRV, many metrics are developed to describe HRV, and they can be classified into three categories: time-domain, frequency-domain, and non-linear metrics (Table 1). These metrics can be obtained from short-term (less than $10 \mathrm{~min}$ ) or long-term ECG recording $(24 \mathrm{~h})$ (Task Force of the European Society of Cardiology the North American Society of Pacing Electrophysiology, 1996).

The time-domain metrics are considered to be the simplest methods to extract, since they are directly based on the normalto-normal (NN) intervals or on the instantaneous HR extracted from the ECG. The time-domain metrics are then calculated through statistical or geometric methods. The statistical methods consist in applying operations, such as mean, standard deviation, or square root, on: the direct $\mathrm{NN}$ interval measurements, thus obtaining metrics, such as standard deviation of RR (SDRR), standard deviation of NN (SDNN), standard deviation of the average NN (SDANN), and standard deviation of NN (SDNN) Index, and the difference between NN intervals, thus obtaining metrics, such as root mean square of consecutive RR interval differences (RMSSD), NN50, pNN50, and pNN20 (Task Force of the European Society of Cardiology the North American Society of Pacing Electrophysiology, 1996; Oliveira et al., 2019b; Patural et al., 2019).

The geometric methods consist in transforming the NN interval measurements into a geometric pattern, such as the sample density distribution of the NN intervals (or their difference), from which metrics, such as the triangular interpolation of the NN (TINN), the HRV Triangular Index, and the HRV Index, can be obtained. A Poincaré plot can also be used to plot each $\mathrm{NN}$ interval in relation to the previous $\mathrm{NN}$ interval and to calculate the standard deviation of the main cluster of data-points, either crosswise (SD1) or lengthwise (SD2) (Task Force of the European Society of Cardiology the North American
Society of Pacing Electrophysiology, 1996; Oliveira et al., 2019b; Patural et al., 2019). From the SD1 and SD2 metrics, other metrics, such as Cardiac Sympathetic Index (CSI) and Cardiac Vagal Index (CVI), could be obtained (Oliveira et al., 2019b).

The frequency-domain metrics derive from the analysis of the ECG power spectrum. The power of several frequency bands, in particular ultra-low-frequency (ULF), very-low-frequency (VLF), low-frequency (LF), and high-frequency (HF), are then extracted, as well as other metrics, such as LF and HF normalized indices (LFn and HFn) and the LF/HF ratio. Another frequency index used is the total power (TP), which represents the global variability (Task Force of the European Society of Cardiology the North American Society of Pacing Electrophysiology, 1996; Patural et al., 2019).

Time- and frequency-domain metrics are, however, limited: they could fail in discriminating among different signals that display similar characteristics, such as the same mean or standard deviation. Moreover, ECG recording can manifest several irregularities in the RR series or complex oscillatory phenomena that linear metrics cannot properly describe. Therefore, nonlinear analyses were developed to better grasp the complexity behind the brain and ANS influences on HRV (Task Force of the European Society of Cardiology the North American Society of Pacing Electrophysiology, 1996; Bravi et al., 2011). Actually, the geometric methods can be considered a kind of non-linear analysis (Task Force of the European Society of Cardiology the North American Society of Pacing Electrophysiology, 1996; Javorka et al., 2017; Patural et al., 2019).

Several studies showed the usefulness of non-linear metrics over linear ones in evaluating the autonomic development (Uhrikova et al., 2015; de Souza Filho et al., 2019; Oliveira et al., 2019b), the interactions between ANS, brain, and stress axis (Thayer and Lane, 2000), and the infant's adaptation capacity (Gonçalves et al., 2017; Shaffer and Ginsberg, 2017; Urfer-Maurer et al., 2018).

Many non-linear metrics can be calculated, and every nonlinear analysis can be performed at different levels of complexity. Examples are: detrended fluctuation analysis (DFA), sample entropy (SampEn), approximate entropy (ApEn), multiscale entropy (MSE), symbolics dynamics, coarse graining spectral 
analysis, fractal analysis, and deceleration and acceleration analyses (Task Force of the European Society of Cardiology the North American Society of Pacing Electrophysiology, 1996; Javorka et al., 2017; Patural et al., 2019).

While linear metrics seem more useful when the HR follows periodic oscillations, non-linear metrics, such as SampEn, ApEn, MSE, DFA, and symbolic dynamics metrics, seem more robust to cardiac recording artifacts (Javorka et al., 2017; Stapelberg et al., 2017). Linear and non-linear metrics can, however, detect complementary physiological behaviors (Task Force of the European Society of Cardiology the North American Society of Pacing Electrophysiology, 1996; Bravi et al., 2011): the HRC index, which contains both, represents the best available evidence of their usefulness (Fairchild and O'Shea, 2010).

Actually, many more different techniques of variability analysis are available. To encompass all of them and to refine the original Task Force classification, further classifications have been proposed. Bravi and colleagues, for example, regrouped the various techniques into five domains based on the information they extrapolate: statistical, geometric, energetic, informational, and invariant (Bravi et al., 2011). The present paper, however, will follow the Task Force classification since it still represents the most used.

Although several HRV metrics have been proved useful, few metrics have a precise physiological and clinical interpretation (Table 2). However, we have to consider two crucial limitations: (1) too often HRV is viewed as influenced by only the ANS, with the tendency of reducing a metric to as simply sympathetic or parasympathetic, and (2) although many studies investigate several metrics, the attention is focused mainly on univariate approaches that try to reduce the variables considered at a minimum. This approach could substantially limit the usefulness of the models applied and, thus, the understanding of HRV physiological nature and clinical usefulness (Bravi et al., 2011; Frasch, 2018).

To overcome these limitations, multivariate analyses were introduced-e.g., continuous individual multiorgan variability analysis (CIMVA) (Seely et al., 2011; Frasch et al., 2014)-, and many researchers started to rely on $\mathrm{ML}$ and $\mathrm{AI}$ algorithms (Semenova et al., 2018; Vassar et al., 2020). As a consequence, research begun to integrate various metrics-as shown by the HeRO monitor, whose HRC index derives from a logistic regression applied on the standard deviation of the RR intervals, sample asymmetry, and SampEn and aims to detect pathological heart decelerations to predict the risk of sepsis within $24 \mathrm{~h}$ (Fairchild and O'Shea, 2010)-, to combine measurements from different organs for predicting clinical conditions (Kumar et al., 2020), and to define models able to describe HRV as a phenomenon influenced by the whole organism (Frasch, 2020).

\section{The HRV as a Window on the ANS}

The ANS plays a central role in homeostasis maintenance and allostatic adaptation, allowing the organism to change its behavior based on the circumstances and stressors, whether internal or external (Thayer and Lane, 2000).
The two ANS branches, the sympathetic (SNS) and parasympathetic system (PNS), continuously modify their balance to finely regulate the organic systems, including the cardiovascular, respiratory, gastroenteric, metabolic, and immune-inflammatory ones (Rees, 2014; Mulkey and du Plessis, 2019). The two ANS branches connect with several brain circuits known as the central autonomic network (CAN), which influences both the ANS and the higher cortical functions, such as cognition control, emotional regulation, and behavior (Benarroch, 1993; Thayer and Lane, 2000).

To cope with the environmental stimuli, the CAN regulates the complex non-linear interaction between SNS and PNS. In the sinoatrial node, this interaction modulates the HRV (Billman, 2011; Shaffer and Ginsberg, 2017). Tools, such as ECG or photoplethysmography (PPG), which monitor cardiovascular activity, can thus evaluate autonomic brain-heart interactions and give information regarding the ANS through HRV metrics (Table 2).

These metrics are usually viewed as revealing different facets of the SNS and PNS activities, with some metrics more related to one of the two branches and the other reflecting more complex ANS, cardiocirculatory, and respiratory activities (Shaffer and Ginsberg, 2017; Oliveira et al., 2019b). However, HRV may reflect much more than the autonomic regulation of cardiac activity. On the one hand, it has been recently discovered, in ovine models, that the fetal heart has already an intrinsic sinoatrial node activity that can affect HRV and that can be affected by adverse conditions (e.g., chronic hypoxia) in the last trimester of pregnancy (Frasch et al., 2020). On the other hand, HRV seems to be greatly influenced by information coming from the whole organism (e.g., the gut or the immune system) through systemic afferent pathways, such as the vagus nerve (Frasch, 2020).

Moreover, HRV may represent an index of the adaptive regulation processes performed by the CAN (Thayer et al., 2009, 2012). Indeed, HRV correlates with CAN activity measured by functional magnetic resonance imaging (Thayer et al., 2012) and several measures of cognitive, emotional, and behavioral regulation (Holzman and Bridgett, 2017; Forte and Casagrande, 2019). Since various HRV metrics showed to be correlated with stress and inflammation, HRV analysis has the potential to give information about the subject's health (Kim et al., 2018; Williams et al., 2019).

During fetal and neonatal life, the ANS undergoes a prolonged process of development and maturation, during which it remains vulnerable to developmental disruption from a variety of stressful physiological and environmental stimuli. Such stimuli-which can occur during pregnancy (e.g., congenital disease, fetal growth restriction, maternal stress/nutritional deficiency), labor (e.g., premature, complicated, or prolonged birth), and even in the NICU environment (e.g., invasive procedures, loud noise, and bright light)—can significantly influence the developmental trajectory of both the ANS and the CAN. They can reduce the newborn's capacity to efficiently adapt to a continually changing and challenging environment (Mulkey and du Plessis, 2019). 
TABLE 2 | Interpretations and/or usefulness of some HRV metrics (Task Force of the European Society of Cardiology the North American Society of Pacing Electrophysiology, 1996; Shaffer et al., 2014; Shaffer and Ginsberg, 2017; Oliveira et al., 2019b).

\begin{tabular}{|c|c|}
\hline Metric & Interpretation \\
\hline \multicolumn{2}{|l|}{ Most common metrics } \\
\hline RMSSD & Main time-domain measure to assess the HRV modulation due to vagal activity \\
\hline SDNN & $\begin{array}{l}\text { Standard metric of overall HRV, influenced by both SNS and PNS. Gold standard for medical stratification of cardiac risk in adults when } \\
\text { recorded over a } 24 \mathrm{~h} \text { period }\end{array}$ \\
\hline ULF & $\begin{array}{l}\text { No consensus regarding the mechanisms underlying ULF power. Very slow-acting biological processes, such as circadian rhythms, are } \\
\text { implicated }\end{array}$ \\
\hline VLF & $\begin{array}{l}\text { Related to the heart's intrinsic nervous system, which generates VLF rhythm when afferent sensory cardiac neurons are stimulated. SNS } \\
\text { activity due to physical and stress responses influences its oscillations amplitude and frequency }\end{array}$ \\
\hline LF & Non-specific index that reflects baroreceptor activity, it contains contributions of both the sympathetic and parasympathetic influences \\
\hline HF & $\begin{array}{l}\text { Expression of parasympathetic activity, it corresponds to the HR variations related to the respiratory cycle known as RSA. It changes } \\
\text { according to vagal modulation but does not reflect vagal tone }\end{array}$ \\
\hline LF/HF & $\begin{array}{l}\text { Used to estimate SNS and PNS balance, although LF does not purely represent SNS, and PNS and SNS interact in a complex non-linear } \\
\text { manner }\end{array}$ \\
\hline \multicolumn{2}{|c|}{ Complex metrics or groups of metrics } \\
\hline fABAS & $\begin{array}{l}\text { Scale used for evaluating fetal ANS maturation. It derives from the integration of Hoyer et al. (2013): } \\
\text { - amplitude (ACTAMP20), evaluating the fluctuation range of heart beat intervals above an approximated baseline increasing complexity; } \\
\text { - skewness, evaluating the complexity of heart rate patterns essentially modulated by complex sympatho-vagal rhythms; } \\
\text { - gMSE(3), evaluating the asymmetry, contribution of vagal and sympathetic activity with their different time constants, decline of } \\
\text { decelerations, and formation of acceleration patterns; } \\
\text { - pNN5, evaluating the formation of vagal rhythms; } \\
\text { - VLF/LF, evaluating the baseline fluctuation in relation to sympatho-vagal modulations. }\end{array}$ \\
\hline $\begin{array}{l}\text { DFA } \alpha 1-\text { Asyml - } \\
\text { KLPE - SDLE } \alpha\end{array}$ & Used for assessing vagal modulation in fetuses (Herry et al., 2019) \\
\hline HRC index & $\begin{array}{l}\text { Displayed by the HeRO monitor to estimate the risk of sepsis within } 24 \mathrm{~h} \text {. It derives from a logistic regression calculated on standard } \\
\text { deviation of the RR intervals, sample asymmetry, and SampEn to detect irregularities and transient decelerations in } \mathrm{HR} \text {. HRC index is higher } \\
\text { in preterm infants than in full term ones (they show less variability), and it decreases as postmenstrual age increases. HRC index can also } \\
\text { rise due to acute inflammation, respiratory deterioration, intraventricular hemorrhage, brain injury, NEC, surgery, ventilation, and drugs, such } \\
\text { as anticholinergics, anesthetics, and dexamethasone (in this last case, HRC decreases) (Fairchild and O'Shea, 2010; Fairchild, 2013; Kumar } \\
\text { et al., 2020) }\end{array}$ \\
\hline $\begin{array}{l}\mathrm{RMS}_{S}-\mathrm{RMS}_{\mathrm{L}}-\mathrm{DFA} \\
\alpha_{S}-\mathrm{LF}-\mathrm{HF}\end{array}$ & $\begin{array}{l}\text { Used to estimate outcomes in case of HIE, especially during hypothermia treatment. Low values of RMS }, R_{S M S}, D F A \alpha_{S} \text {, and LF and a } \\
\text { high value of HF may predict an adverse outcome and the need of adjuvant neuroprotective therapies. These metrics could discriminate } \\
\text { among different types of brain injury. All these metrics decreased also proportionally to NEC severity (Metzler et al., 2017; Al-Shargabi et al., } \\
\text { 2018; Campbell et al., 2018) }\end{array}$ \\
\hline
\end{tabular}

It is thus essential to know how ANS develops during pregnancy and after birth, and how HRV changes as a result, in order to make efficient use of HRV as an assessment tool.

\section{The ANS Development and Its Relationship With HRV}

Fetal cardiac activity is usually measured through cardiotocography (CTG), which can monitor fetal HR via ultrasound waves (Ayres-de-Campos and Bernardes, 2010). Although able to detect HR accelerations and decelerations, this tool poorly discriminates QRS complexes and is unsuitable to measure fetal HRV (fHRV), especially short-term HRV (Van Leeuwen et al., 2014). Therefore, in the last 20 years, researchers have relied on fetal magnetocardiography (fMCG) and ECG (fECG) to monitor fHRV and assess its correlation with ANS development (Hoyer et al., 2013, 2015).

fECG and fMCG use electrophysiological mechanisms to monitor cardiac activity and better discriminate QRS complexes (Hoyer et al., 2017). fMCG, which is obtained through the spatiotemporal measurement of the heart-related magnetic field, allows a stable and precise HRV assessment during the second and third pregnancy trimesters. However, fMCG is available in a few centers worldwide due to its high cost (Hoyer et al., 2017). fECG can be obtained through electrodes placed on the mother's abdomen (Hoyer et al., 2017) or directly on the fetal cranium during labor (Warmerdam et al., 2016). fECG performs less accurate measurement and, between 28 and 32 weeks gestational age (GA), is less reliable since the fetus is almost entirely covered by the vernix caseosa, which isolates the fetus hindering the recording (Hoyer et al., 2017). Besides, fECG shows a considerable signal loss $(30 \pm 24 \%)$ with $3.6 \pm 1.7$ gaps $/ \mathrm{min}$, which limits shortterm and entropy-related HRV metrics measurements. As a result, fECG shows higher values for these metrics than fMCG (Van Leeuwen et al., 2014).

Using fMCG, Hoyer and colleagues defined the fetal autonomic brain age score (fABAS) (Table 2), which derives from a multivariate analysis of five different metrics-amplitude, gMSE(3), skewness, pNN5, and VLF/LF-and managed to assess ANS development from the 22nd week GA (Hoyer et al., $2013,2015)$. Other authors have then proposed to add other metrics to fABAS to define more complete and precise models (Schmidt et al., 2018). 
Applying regression models on metrics grouped in shortterm and long-term amplitude fluctuations (AMP), complexity (COMP) and pattern categories, Hoyer et al. found that ACst1, STV, MSE4, and skewness were the metrics that best correlated with fetal maturation (Hoyer et al., 2019). During the third trimester, AMP, COMP, and pattern metrics increase, although with different timing (Hoyer et al., 2019), together with sympathetic and parasympathetic modulations. The baseline HR also becomes more regular and with a more stable rhythm (Schneider et al., 2018). As GA increases, even linear metrics-RMSSD, SDNN, LF, and HF-rise as well. These changes indicate the ANS development and better fetal regulatory capacities (Hoyer et al., 2009; Cardoso et al., 2017; Aye et al., 2018).

To achieve an optimal maturation, the ANS, in particular the PNS, needs 37 weeks GA (Patural et al., 2019): indeed, preterm newborns show a sympathetic predominance (Yiallourou et al., 2012). The PNS begins to develop during the first trimester, when the lateral hypothalamus differentiates and the vagus nerve myelination increases (Koutcherov et al., 2003; Cheng et al., 2004). From the 32nd week GA, the vagus nerve regulatory activity increases with the appearance of the baroreflex mechanisms and the respiratory sinus arrhythmia (RSA) - the capacity of HR regulation based on respiratory rate. Meanwhile, fetal movements and HR increase indicate a rise in the SNS activity (DiPietro et al., 1996). The ANS regulatory function, especially the parasympathetic, develops until birth when the ANS has to react to the new environmental conditions by adapting the cardiovascular and respiratory systems during the fetal-neonatal transition (Mulkey and du Plessis, 2018).

At birth, the ANS shows a slight sympathetic predominancehigh LF and low HF-, but in the first $24 \mathrm{~h}$ after birth, several metrics (e.g., HR, SDNN, CSI, HFn, LFn, TP, SD2, HRV Index, and Parseval Index) change significantly (Oliveira et al., 2019b). In particular, during the first $12 \mathrm{~h}$, the PNS gradually begins to take over, as shown by the LF/HF ratio reduction (Patural et al., 2019).

During the first days and weeks of life, both preterm and term newborns show an increase in RMSSD, SDNN, and HF, although preterm newborns show lower HRV than term ones at the same postmenstrual age: they display lower values of RMSSD, LF, and HF and a higher LF/HF ratio, which likely reflect an ANS that needs more time to mature (Lucchini et al., 2016; Cardoso et al., 2017; Aye et al., 2018).

The ANS, in particular the PNS, continues to develop during the first 2 years of life considerably: indeed, every time, frequency, geometric, and non-linear metric tend to increase (Patural et al., 2019).

Since many HRV metrics change during the ANS development, several authors investigated if specific correlations could be established between those changes and the fetal health status.

According to Hoyer et al. (2019), some AMP and COMP metrics correlate with maternal lifestyle (smoking and physical activity), intrauterine growth restriction (IUGR), and gestational diabetes. Despite the maternal and fetal circulation being distinct systems, HRV fluctuations could represent a coupling mechanism between the two: indeed, maternal HR, TP, and HF correlate with some fHRV metrics, especially during active sleep and when GA > 32 weeks. In particular, maternal HR correlates positively with fHR and negatively with fHRV (Zöllkau et al., 2019).

A different approach based on coupling maternal and fetal HR through phase rectified signal averaging (PRSA) analysis found a correlation between the two HRs, but only for stressed mothers (Lobmaier et al., 2020). A correlation between some fHRV metrics and maternal TP and RMSSD was also found in normotensive mothers, whereas in preeclamptic pregnancies, there was no correlation despite substantial reduction in both maternal HRV (SDNN, RMSSD, pNN50, TP, VLF, LF, HF) and fHRV metrics (SDNN, RMSSD, pNN50, TP, VLF, LF, HF, STV, LTV, $\mathrm{N}^{\circ}$ of accelerations) (Lakhno, 2017).

During labor, although uterine contractions may alter fHRV recording, fetal scalp electrode (FSE) can measure fHRV with adequate precision and give more accurate information about fetal distress than traditional CTG, thus better discriminating between healthy fetuses and those with acidosis (Gonçalves et al., 2006; van Laar et al., 2010, 2011; Spilka et al., 2012; Abry et al., 2013; Georgieva et al., 2013). pH levels during labor were correlated with both linear (Gonçalves et al., 2006; van Laar et al., 2010) and non-linear fHRV metrics (Li et al., 2005; Ferrario et al., 2006; Abry et al., 2013; Chudacek et al., 2014; Signorini et al., 2014), and the combination of linear and non-linear metrics seemed to detect acidosis more precisely than the two types of metrics alone (Spilka et al., 2012; Warmerdam et al., 2018). In addition to FSE, transabdominal fECG with a sampling frequency of $900 \mathrm{~Hz}-$ way higher than the frequency of $4 \mathrm{~Hz}$ used for CTG-may efficiently measure fHRV during labor and predict neonatal $\mathrm{pH}$ level and acid-base balance, especially through CIMVA (Frasch et al., 2014; Li et al., 2015). These results are of great significance since acid-base imbalance can be correlated to brain neuroinflammation and, therefore, to the risk of developing brain injury (Xu et al., 2014).

Together with continuous fHRV assessment, the selective measurement and comparison among the fHRV metrics obtained during labor contractions and the ones obtained between successive contractions managed to better detect fetal distress (Warmerdam et al., 2018). Several fHRV metrics increased during contractions and decreased during rest periods (Romano et al., 2006; Cesarelli et al., 2010; Warmerdam et al., 2016), and this variability was lower in fetuses with acidosis than in healthy ones, pointing at a lower cardiovascular adaptation capacity (Warmerdam et al., 2016). Entropy metrics, such as SampEn and ApEn, could also detect fetal distress from the 30th week GA (Ferrario et al., 2006). These two metrics, together with time-domain, complexity (e.g., Lempel Ziv complexity), and PRSA-derived ones, could also distinguish between fetuses with and without IUGR (Signorini et al., 2014).

Non-linear metrics could explain why male fetuses show higher HRV than female but are at higher risk of comorbidity 
(DiPietro and Voegtline, 2017): while linear metrics are higher in male newborns, non-linear metrics that evaluate entropy are higher in female newborns (Gonçalves et al., 2017; Spyridou et al., 2018). Moreover, non-linear metrics, such as ApEn or MSE, could describe better than linear metrics the ANS development that happens around the last weeks of gestation, when the fetus prepares for birth (Spyridou et al., 2018).

Interesting results came also from animal models of fetal inflammation. Studies on models of hypoxic-ischemic events in ovine fetuses showed that many fHRV metrics changed in the hours after the event: VLF decreased, whereas SampEn, HF, and RMSSD increased (Frasch et al., 2016; Kasai et al., 2019). Besides, the rise in RMSSD correlated positively with interleukin (IL)-1 $\beta$ levels (Frasch et al., 2016), whereas lipopolysaccharides administration showed that multidimensional fHRV metrics (i.e., both linear and non-linear) could predict the fetal inflammatory response (Durosier et al., 2015; Herry et al., 2016).

In an ovine model of repetitive umbilical cord occlusions, PRSA applied to fHRV detected hypoxic events and distinguished between mild, moderate, and severe hypoxia-acidemia (Rivolta et al., 2014). This result was deepened in another study where an ML algorithm based on RMSSD time series sampled at $1,000 \mathrm{~Hz}$ was developed: after $2 \mathrm{~h}$ of training with the individual fHRV during labor, the algorithm predicted fetal cardiovascular decompensation with $92 \%$ sensitivity, $86 \%$ accuracy, and $92 \%$ precision. When using $4 \mathrm{~Hz}$ CTG, the algorithm showed a $67 \%$ sensitivity, $14 \%$ accuracy, and $18 \%$ precision-that is, sample frequency really matters (Gold et al., 2019).

fHRV assessment could thus give precious information both on the physiological fetal maturation and on the occurrence of pathologies that, whenever left unresolved, are able to induce lifelong complications (Hoyer et al., 2017; Frasch, 2018). Assessing HRV in newborns and infants could, thus, greatly improve neonatal care (Kumar et al., 2020). Therefore, a discussion is required about the technology used to measure HRV in newborns and infants and about the physiological or environmental factors that could influence HRV.

\section{A TECHNOLOGICAL ANALYSIS TO EFFICIENTLY MONITOR AND USE HRV}

\section{The Current NICU Monitoring Instruments}

The NICU incubators are closed devices equipped with advanced devices to monitor the newborn's conditions. In essence, they guarantee a clean and controlled environment concerning temperature, humidity, and even mechanical noise and light. They monitor vital signs through sensors and electrodes placed on the babies (Rajalakshmi et al., 2019).

The foremost vital signs-temperature, blood pressure, pulse frequency, $\mathrm{HR}$, and $\mathrm{SpO}_{2}$-are measured through monitors for the control of pressure, cardiorespiratory system, skin temperature, and carbon dioxide and oxygen concentrations; pulse oximetry; and mechanical ventilator. In the case of anomalies, an alarm alerts the NICU staff to intervene promptly (Rajalakshmi et al., 2019).

ECG represents the gold standard to monitor HR calculated through the RR interval (Phillipos et al., 2016; Alonzo et al., 2018). Pulse oximetry can instead measure HR through the pulse rate, but its usefulness is debated: evaluating pulse rate is difficult when peripheral tissue perfusion is reduced (Mizumoto et al., 2012), and the measurement is sensitive to motion artifacts (Sahni et al., 2003).

Pulse oximetry performs a simple, non-invasive, and accurate $\mathrm{SpO}_{2}$ measurement to evaluate the risk of hypoxia and/or hyperoxemia, the appropriateness of the oxygen administered to the newborn, and the presence of ductal dependent congenital cardiac diseases (Stenson, 2016; Kumar et al., 2020). Pulse oximetry, however, struggles to detect hyperoxemia when $\mathrm{SpO}_{2}>94 \%$ (Hay, 1987).

Blood pressure (influenced by cardiac output and peripheral vascular resistance) can be measured through an external cuff, which, however, can underestimate it in hypotensive or pathological preterm newborns (Cunningham et al., 1999). A peripheral arterial catheter can continuously monitor blood pressure, although it can induce severe cardiovascular complications (Werther et al., 2018).

The respiratory rate is calculated through two electrodes placed on the thorax and the abdomen that measure the thoracic impedance changes. Plethysmography gives a more reliable measure but requires a higher number of thoracic electrodes (Weese-Mayer et al., 2000).

Temperature is continuously monitored because preterm newborns struggle to regulate it and can suffer from hypothermia. Incubators can assess the newborn's temperature through skin sensors and probes or infrared thermography, which has the advantage of being contactless and non-invasive, and may adapt the environmental temperature to the newborn's skin temperature (Verklan and Walden, 2014). Due to the sensitive skin of newborns, cutaneous sensors could create stress and even damage the skin (Topalidou et al., 2019).

Despite continuous monitoring to assure high care standards, much of this collected information remains unused. This situation is worth noting since integrating these vital signs could improve the predictive power of the NICU monitors and better help reduce infants' mortality (Sullivan et al., 2018; Joshi et al., 2020; Kumar et al., 2020).

Besides monitoring the infant's clinical condition, incorporating the infant's history would also be of the utmost importance. Information about the baby's fetal health, labor, and past adverse events (e.g., brain injuries) could augment the interpretation of the aforementioned vital signs. In fact, different pathologies in different babies could induce similar alterations in vital signs, and the knowledge of the infant's history could help recognize what is happening (Kumar et al., 2020).

In this difficult task, NICU monitors based on ML algorithms could be useful for clinicians: integrating the infant's past and present data, they could discriminate among the several pathologies that could affect the infant, thus pointing at the best preventive and therapeutic strategy. ML algorithms have 
already helped in predicting the risk of various conditions (e.g., cerebral hemorrhage and hyperbilirubinemia) and in reducing false alarms, thus improving neonatal care (Daunhawer et al., 2019; Malacova et al., 2020; Ostojic et al., 2020; Turova et al., 2020; Vassar et al., 2020).

\section{The Current State of HRV Assessment and Limitations}

The gold standard to evaluate HRV consists of specific calculations on the electrocardiographic HR registrations (time elapsed between two adjacent $\mathrm{R}$ peaks) in term and preterm newborns (Kevat et al., 2017).

However, ECG does not seem to be always used properly, and as a consequence, HRV measurement may suffer from methodological issues: for instance, the NICU tools used to monitor HR may have a low sampling frequency $(<200 \mathrm{~Hz})$, making the HRV analysis unreliable under several circumstances (e.g., patients with low RR variability due to heart failure), in particular for frequency and non-linear metrics. Some authors indeed advised to use a sampling frequency of at least $500 \mathrm{~Hz}$ (Laborde et al., 2017; Shaffer and Ginsberg, 2017). Moreover, in the research field, there is the tendency to use proprietary software without specifying the exact algorithms used to extract the HRV parameters and, thus, limiting the usefulness of the results (Heathers, 2012; Pagani et al., 2012).

Particularly interesting is the case of the frequency-domain metrics, LF and HF. Despite being widely used, the frequency threshold of $0.15 \mathrm{~Hz}$ is used to discriminate between them in adults derived from animals, and it seems that it has never been validated in humans (Hayano and Yuda, 2019). Therefore, the frequency-domain metrics could be biased from the beginning: this could be a reason why LF and HF do not reflect a specific physiologic function, but the interaction between PNS, SNS, and respiratory activity (Hayano and Yuda, 2019). Moreover, not all studies on newborns use the same thresholds for discriminating between LF and HF (Andersen et al., 2019).

Among the several HRV metrics (Table 1), few of them have been validated (Table 2; Shaffer and Ginsberg, 2017; Hayano and Yuda, 2019), especially in newborns and infants (Cardoso et al., 2017; Oliveira et al., 2019b). For several metrics, their significance can also change according to the measurement duration: short (about $5 \mathrm{~min}$ ) or long term (until $24 \mathrm{~h}$ ) (Pernice et al., 2019b).

Therefore, standardizing how researchers and clinicians should monitor HRV and calculate their metrics is essential. A uniform methodology could also help in defining normative HRV values for infants (stratified for their age and other fetal development characteristics)-during the years, several studies attempted to fill this gap (Clairambault et al., 1992; Eiselt et al., 1993; Spassov et al., 1994; Cardoso et al., 2017; Schneider et al., 2018; Oliveira et al., 2019b; Patural et al., 2019; Shuffrey et al., 2019) - to promptly recognize even deadly complications, but results are still controversial.

As with the integration of the baby's vital signs and history, clinicians could rely on ML and AI to overcome this difficulty. ML could both define normative HRV values for single or complex metrics and even create models able to predict how the newborn's condition will evolve based on big-data analysis (Fairchild and Aschner, 2012; Kumar et al., 2020). Indeed, in several research fields including neonatology, neurology, and cardiology, advanced ML is being successfully used to detect specific pathophysiological characteristics (Fraser, 2017; Pinaya et al., 2019; Tu et al., 2020; Turova et al., 2020; Vassar et al., 2020).

\section{The Use of PPG to Measure HRV as an Alternative to ECG}

In the last years, a new methodology that uses PPG has been applied to measure the peripheral pulse rate variability (PRV) through sensors placed on the fingers (Blackford et al., 2016; Choi and Shin, 2017). The growing interest toward PPG rests on its simplicity, low-cost, safety, and minimal invasiveness and on its capacity to assess signs, such as oxygen saturation, and to extract cardiorespiratory parameters (Pernice et al., 2018, 2019a; Singh et al., 2018).

PPG is an optical measurement technology used to detect blood volume changes in peripheral capillaries. It needs a light source and a photodetector that can measure the light intensity variations related to the capillary perfusion changes. PPG is used in physiological and clinical research to measure blood pressure variability (McDuff et al., 2018; Pernice et al., 2018, 2019b).

The absence of electrodes helps reduce the newborn's stress and pain. It may also decrease the risk of limb ischemia and skin lesions due to electrode-related irritation and, thus, the risk of skin infections with subsequent use of antibiotics and disinfectants, which can alter the cerebral pressure and perfusion when inhaled (Blanik et al., 2016; Zhao et al., 2016; Cobos-Torres et al., 2018). ECG alternatives to monitor HRV are paramount since, due to altered evaporation of body water, electrodes on large skin areas may influence the balance between heat and water in preterm newborns who weigh less than $1,000 \mathrm{~g}$ (Blanik et al., 2016). Avoiding electrodes would also reduce the workload of NICU professionals since they might avoid controlling the correct positioning of patches (Cobos-Torres et al., 2018).

From a PPG signal, a PRV time series can be registered and analyzed similarly to HRV (Pernice et al., 2018). However, HRV and PRV are different: HRV is calculated from the cardiac electrical activity, whereas PRV from the mechanics of pulsatile blood. PPG and ECG also show different waveforms: they are differently influenced by the sampling frequency (Choi and Shin, 2017).

PPG recording is affected by physiological factors related to the pulse wave transmission along the vascular bed and by measurement errors, such as artifacts due to movements. In the last years, several authors have attempted to reduce or eliminate these errors that can make PRV and HRV disagree (Pernice et al., 2019b). The PRV analysis should also use a correct sampling frequency to obtain measurements concordant with ECG (Choi and Shin, 2017; Hejjel, 2017).

Some authors found that, for several metrics (SDNN, SDSD, RMSSD, NN50, pNN50, TP, HF, LF/HF, LFn, and HFn), a frequency as low as $25 \mathrm{~Hz}$ could give comparable results to those obtained with a $10 \mathrm{kHz}$-sampled ECG in healthy subjects (Choi and Shin, 2017). However, very low sample frequency 
could not correctly detect the effect of vagal modulation on HRV/PRV (as it is associated with high frequency), making frequency and non-linear metrics unreliable-time-domain metrics appear instead to be more robust (Choi and Shin, 2017; Béres et al., 2019). Notwithstanding this, other studies used a sample frequency of $200 \mathrm{~Hz}$ obtaining reliable results (Sun et al., 2012; Elgendi et al., 2016). Thus it can be argued that the above-mentioned recommendations for ECG could also be valid for PPG.

Most studies consider PRV and HRV to be exchangeable (Blackford et al., 2016; Pernice et al., 2018; Singh et al., 2018), although sometimes different measurements were detected when people (mostly adults) were under postural $\left(45^{\circ}\right.$ head-up tilt test) or mental (arithmetic test) stress, especially for RMSSD, LF, HF, and LF/HF (Pernice et al., 2019b).

In the context of NICU, PPG provides accurate data in addition to the ease of use. A recent study showed that, through specific algorithms, PPG could detect the movement artifacts, remove them from the recordings, and use them to evaluate the onset and duration of the newborn's movements: this measurement could help assess the motor development and even the cognitive growth related to motor control (Zuzarte et al., 2019). These elements could favor the use of PPG over other devices. However, some studies showed lack of sufficient data sampling to perform adequate statistical analysis, calling, therefore, for more robust data collection (Kevat et al., 2017; Henry et al., 2020).

In the last years, non-contact video-photoplethysmography (vPPG) has been introduced: a non-invasive optical technology able to remotely detect the blood volume changes, using natural light. A digital camera measures the little cutaneous light intensity changes, which derive from the cardiovascular rhythm of the skin blood perfusion (Blanik et al., 2016; Valenza et al., 2018). vPPG could accurately monitor the newborn's movements (Cobos-Torres et al., 2018) and even help prevent sudden infant death syndrome (SIDS) (Zhao et al., 2016).

Since HR tends to significantly decrease over minutes or hours before SIDS occurrence, a technology that could continuously monitor $\mathrm{HR}$, in particular, during night-time or with low ambient light-SIDS happens especially in these conditionscould efficiently prevent SIDS. Using specific algorithms, vPPG seems to make accurate HR measurements during night-time; besides, since vPPG does not rely on skin sensors, it could avoid fatal errors linked to their detachment due to the baby's movements (Zhao et al., 2016).

From the available evidence on newborns and adults, vPPG and ECG would seem to agree in the detection of $\mathrm{HR}$ and several HRV metrics (e.g., LF, HF, SDNN, SampEn, and Lyapunov exponent): the statistical analyses (e.g., Bland-Altman tests and Pearson's r) showed high correlations, and the measurement errors between the two technologies were similar to those between ECG and other technologies, such as pulse oximeter (Blanik et al., 2014; Villarroel et al., 2014; Blackford et al., 2016; Valenza et al., 2018). Other studies found vPPG and PPG to give similar measurements in the assessment of $\mathrm{HR}, \mathrm{LF}$, and HF: since PPG is usually considered as reliable as ECG, the authors inferred that vPPG could also agree with ECG (Sun et al.,
2012; Aarts et al., 2013; McDuff et al., 2014; Paul et al., 2020). However, more studies on newborns are needed to improve vPPG and to better evaluate its usefulness (Cobos-Torres et al., 2018; Valenza et al., 2018).

Before proceeding further, we briefly mention ballistography (BSG), another contactless and unobtrusive technology that could help HRV assessment. BSG can detect the mechanical forces exerted by the body, including body movements, breathing motion, and heartbeat, through sensors placed in the bedding or mattress under the infant's body or even in the incubator rack. From the mechanical signals recorded by the sensors (e.g., including electromechanical film sensors, loadcells, and accelerometers), BSG successfully extrapolated reliable respiratory waveforms and $\mathrm{HR}$ recordings in preterm newborns (Nukaya et al., 2014; Lee et al., 2016; Joshi et al., 2018, 2019a). While in neonatology, BSG represents a new technology that should be improved to optimally and precisely detect the weak forces of the baby's body; in adults, BSG gave reliable HRV measurements as compared with ECG (Shin et al., 2011; Wang et al., 2015).

\section{Factors That Can Alter HRV Assessment}

To optimally use HRV, NICU professionals must be aware there are factors that can influence HRV recording, including the sleep stage, the infant's position, and the NICU/incubator environment (De Jonckheere and Storme, 2019; Weber and Harrison, 2019).

HRV in newborns is often measured during sleep, which shows two main stages: the quiet sleep (QS), characterized by the absence of movements and slow rhythmic breathing, and the active sleep (AS), characterized by myoclonic twitching, facial, eye, and head movements, and irregular heart and breath rates (Walusinski, 2006; Dereymaeker et al., 2017).

AS and QS show, respectively, a sympathetic and a parasympathetic predominance (Frasch et al., 2007; Yiallourou et al., 2012), with HRV metrics, such as SDNN, LF/HF, LF, and TP higher in AS than in QS. Sometimes, parasympathetic-related metrics were found to be higher during AS than QS (although by a lower degree than the sympathetic-related metrics), maybe to balance the sympathetic activity (Yiallourou et al., 2012; StéphanBlanchard et al., 2013; Fyfe et al., 2015; Thiriez et al., 2015; Schneider et al., 2018).

If this were the case, altered parasympathetic-related metrics during AS could reveal that the vagal system and the ANS still have to fully develop (Yiallourou et al., 2012). An immature PNS may, thus, fail to balance the SNS and the hypothalamicpituitary-adrenal axis activity under stressful conditions, thus increasing the risk of sepsis, necrotizing enterocolitis (NEC), or even SIDS (Yiallourou et al., 2012; Stone et al., 2013; Sullivan et al., 2014; Mulkey et al., 2018).

Indeed, healthy newborns show an increase in several metrics during a head-up tilt test, thus recruiting both ANS branches to counter the hypotensive stressor (Yiallourou et al., 2012). Besides, newborns who later displayed abnormalities, such as altered neurological development (i.e., cerebral palsy, language or mental retardation, vision or hearing disability, or attention disorders) showed altered HRV (lower total and non-harmonic power) just during AS (Thiriez et al., 2015). 
Although the studies do not perfectly agree (Fyfe et al., 2015), the prone position correlates with lower SDRR and RMSSD (Galland et al., 2006; Lucchini et al., 2016) and also lower sympathetic tone measured through LF and TP (Jean-Louis et al., 2004): these results could indicate an altered ANS response, possibly clarifying the higher risk of SIDS during prone sleep (Elhaik, 2016). The side position seems to stabilize HR and prevent oxygen desaturation during feeding in newborns with GA $<34$ weeks (Thoyre et al., 2012), although a recent study failed to find the same results (Raczyńska and Gulczyńska, 2019).

Concerning the NICU environment, temperature, light, and noise could be a significant source of stress that can affect infants' HRV (De Jonckheere and Storme, 2019; Weber and Harrison, 2019).

Warm incubators correlated with higher HR, lower parasympathetic activity, HF, and shorter RR intervals (Franco et al., 2000). When the incubator temperature was $2^{\circ} \mathrm{C}$ lower than the newborn's skin temperature, the parasympathetic-related metric RMSSD increased in all sleep stages, together with SDNN and HF, whereas the sympathetic-related metric CSI decreased; the opposite findings were obtained when the incubator was $2^{\circ} \mathrm{C}$ warmer (Stéphan-Blanchard et al., 2013). Therapeutic hypothermia in case of hypoxic-ischemic encephalopathy (HIE) could induce an increase in LF, root mean square from detrended fluctuation analysis, which describes shortterm fluctuations $\left(\mathrm{RMS}_{\mathrm{S}}\right)$, root mean square from detrended fluctuation analysis, which describes long-term fluctuations $\left(\mathrm{RMS}_{\mathrm{L}}\right)$, and DFA $\alpha_{\mathrm{S}}$ (Massaro et al., 2017). Extreme variations of environmental or core temperature in both directions can, lastly, destabilize the ANS (as revealed by HRV alteration) and induce possible complications (Fox and Matthews, 1989; Mowery et al., 2011).

Light and sound can alter the newborn's cardiorespiratory functions, increase stress level (Williams et al., 2009; Ozawa et al., 2010; Weber and Harrison, 2019), and modify HRV. Reducing light exposure through eye-mask and covering incubators/cribs can induce a QS with a stable respiratory rate (Shiroiwa et al., 1986; Venkataraman et al., 2018). For sound regulation, positive stimuli, such as human voice, rather than forcefully excluding stressful events, may represent a better option (Weber and Harrison, 2019). The use of tools, such as earmuffs, showed ambiguous results: some studies revealed higher oxygen saturation and quieter sleep, whereas other studies proved higher stress and lower HF (Zahr and de Traversay, 1995; Aita et al., 2013; Almadhoob and Ohlsson, 2020).

The incubator can influence newborns' HRV also through the emission of electromagnetic fields, which indeed correlate with adverse health consequences in infancy (Li et al., 2012). When the incubator power is turned on, LF/HF increases, whereas HF decreases (Bellieni et al., 2008). Positioning the newborn as far away as possible from the incubator power can limit and reverse these effects (Bellieni et al., 2008; Passi et al., 2017).

From the HeRO experience, we also know that some drugs (i.e., dexamethasone, paralytics, anesthetics, and anticholinergics), surgery, and initiation of mechanical ventilation can significantly alter various HRV metrics (Fairchild and O'Shea, 2010; Fairchild, 2013).
If all these factors can impact HRV, thereby biasing its measurement, a real-time monitor could overcome these biases by recording how HRV changes before, during, and after the occurrence of these factors. Again, ML algorithms could help cope with these confounding factors: by weighting and integrating their effects, such technology could help reduce false alarms, exactly as those algorithms that can detect artifacts due to baby's movements (Ostojic et al., 2020).

\section{The Clinical Usefulness of Real-Time HRV to Monitor Newborns}

As with $\mathrm{HR}$ and $\mathrm{SpO}_{2}$, HRV could be monitored in real-time to provide information about the infant's current conditions.

Real-time HRV helped identify stress-related behaviors, which are difficult to visually recognize: for example, LF increased during stress behaviors, whereas HF increased with self-consoling behaviors (Gardner et al., 2018). Moreover, real-time HRV monitoring-as revealed by changes in short- and long-term metrics, such as RMSSD, LF/HF, SampEn, and DFA $\alpha 1$ (but also SDNN, LF, HF, SD1, SD2, ApEn, and DFA $\alpha 2$ ) or in the newborn infant parasympathetic evaluation (NIPE) - correlated with nociceptive events, such as heel stick (Weissman et al., 2012; Butruille et al., 2015).

Derived from short-term HRV metrics related to PNS and reflecting HFn variations, the NIPE aims to assess in realtime the level of the newborn's comfort and nociception (Butruille et al., 2015). Although it failed to correlate with pain scales measuring acute neonatal pain (Cremillieux et al., 2018) and with hemodynamic change following endotracheal intubation in children aged 1-24 months (Zhang et al., 2019), the NIPE succeeded in evaluating prolonged neonatal pain (Buyuktiryaki et al., 2018) and the balance between nociception and antinociception (Weber et al., 2019; Zhang et al., 2019).

Studies that continuously recorded HRV for more than $24 \mathrm{~h}$, or that reviewed those recordings if available, led to discovering that HRV alteration can precede adverse outcomes even by $24 \mathrm{~h}$ (Griffin and Moorman, 2001; Stone et al., 2013; Kumar et al., 2020). Many of the studies about the predictive power of realtime HRV, in particular, in case of infections, relied on the HeRO monitor (Andersen et al., 2019; Kumar et al., 2020). The NIPE technology represents another attempt of introducing real-time HRV monitoring in NICUs, but it needs further evidence (Weber et al., 2019; Zhang et al., 2019).

It was revealed that HRV measured with the HRC index decreased $6 \mathrm{~h}$ before medical NEC and even $16 \mathrm{~h}$ before surgical NEC, thus also indicating the severity of the newborn's condition (Stone et al., 2013). Abnormal heart rate characteristics (HRC) correlated with a several-fold increase in the risk of sepsis, urinary tract infections, and death in the day and week next to alteration (Griffin et al., 2005), and it could also precede the occurrence of SIDS (Zhao et al., 2016). In infants aged 28-35 weeks, low HF (less than $4.68 \mathrm{~ms}^{2}$ ) was found to correlate with the incidence of NEC stage 2+ (Doheny et al., 2014).

In a recent study, HRV combined with respiratory variability analysis changed significantly during the $24 \mathrm{~h}$ before the diagnosis of late-onset sepsis, especially in the last $6 \mathrm{~h}$ where real-time 
monitoring showed signals of cardiorespiratory instability. The reduction in RMSSD, accompanied by a decrease in average acceleration response (AAR, a robust index evaluating the heart capacity to increase its rhythm) and an increase in respiratory variability-related ApEn, predicted late-onset sepsis the most (Joshi et al., 2020).

The HeRO monitor, allowing NICU professionals to continuously observe the HR characteristics variation, reduced relative mortality due to sepsis even more than 20\% (Fairchild, 2013; Kumar et al., 2020), especially when birth weight was lower than 1,000 g (Moorman et al., 2011).

A system that assesses real-time HRV can thus have significant clinical implications. Such a system could display both shortand long-term HRV metrics, thus augmenting HRV predictive usefulness (Voss et al., 2013) and overcoming the limits of shorttime metrics, which are highly sensitive to artifacts (Stapelberg et al., 2017). Due to being non-invasive, real-time HRV would also be preferable to other assessment procedures that can cause discomfort to newborns and take time to be performed (De Jonckheere and Storme, 2019).

However, what could be the best way to make HRV an innovative tool that could augment the newborn's management?

\section{THE FUTURE PERSPECTIVES FOR THE CLINICAL USE OF HRV IN THE NICU}

\section{Technological Innovation to Monitor HRV in Real-Time}

HRV monitoring is more accessible and less invasive than other diagnostic and predicting methods, such as blood sampling for measuring inflammatory cytokines and predicting sepsis (Fairchild, 2013). Real-time HRV monitoring could, thus, represent a significant step forward to improve baby care, especially when clinical decision-making is based on the sole experience of NICU professionals (Mowery et al., 2011; Oliveira et al., 2019a; Weber and Harrison, 2019).

Relying upon the instruments already present in NICUs is paramount (Rajalakshmi et al., 2019); in particular, due to the advantages over ECG, PPG could be the instrument to choose, although PPG-based computation can suffer from precision issues compared with ECG-based computation (Kevat et al., 2017; Pernice et al., 2019b; Henry et al., 2020).

For the sake of simplicity and for attracting clinicians to the use of HRV (King, 2020), the first step could be introducing in NICUs a PRV/HRV monitor that shows a single metric able to correlate with the infants' clinical conditions or development. Although the literature shows that the combination of more metrics can perform better than the use of single metrics (as detailed in previous sections), the literature also shows that single metrics can display good performance (more details in the next sections).

In infants, RMSSD correlated with several outcomes in both the short and the long term: ANS stage of development in the case of prematurity (Hoyer et al., 2009; Lucchini et al.,
2016; Aye et al., 2018; Schneider et al., 2018), late-onset sepsis (Joshi et al., 2020), extubation success (Latremouille et al., 2018), recovering after admission in intensive care units (Marsillio et al., 2019), pain behavior (Weissman et al., 2012), short-term neurological development when measured during low blood pressure episodes (Semenova et al., 2018), and later neurological development at 2 years (Dimitrijević et al., 2016). RMSSD showed also to change according to the incubator temperature, thus revealing how infant's HRV changes due to environmental temperature (Stéphan-Blanchard et al., 2013).

RMSSD seems a reliable index of HRV modulation by vagal activity (Shaffer and Ginsberg, 2017) and has good statistical properties (Task Force of the European Society of Cardiology the North American Society of Pacing Electrophysiology, 1996), and in adult studies, it was associated with adult health more than immune, inflammatory, and metabolic blood markers (Jarczok et al., 2015).

The good relationship with vagal activity makes RMSSD particularly interesting for neonatologists, for three main reasons: (a) the PNS develops during the last weeks of gestation-when preterm birth can occur-and after birth (Schneider et al., 2018; Patural et al., 2019), (b) the PNS activity correlates with the infant's conditions and development (Mulkey and du Plessis, 2019), and (c) since vagal activity correlates positively with inflammation regulation (Fairchild and O'Shea, 2010), RMSSD could help detect newborn's inflammation (as shown in animal fetuses, Frasch et al., 2016). The relation of RMSSD with vagal-related HRV modulation could help clinician in easily interpreting RMSSD changes: the lower the RMSSD, the lower the modulation by the vagal activity, and thus, the worse the infant's conditions.

As a time-domain metric, RMSSD has the advantage over frequency-domain metrics of being a more "direct" measure: RMSSD is obtained by merely calculating the root mean square value of successive time differences between heartbeats (Shaffer and Ginsberg, 2017). Hence, it does not suffer from the conceptual biases that could arise with frequency-domain metrics due to choosing a frequency threshold not validated in humans (Hayano and Yuda, 2019).

Therefore, the NICU real-time monitor could report RMSSD tracking in the previous $24 \mathrm{~h}, 4-5 \mathrm{~min}$, and also 10-30 sultra-short RMSSD recordings are as reliable as short ones (Munoz et al., 2015). The monitor could be equipped with an alarm that activates when the actual RMSSD value changes over a threshold from the newborn's past mean value. The alarm could activate when RMSSD decreases-lower modulation by vagal activity and, thus, risk of worse outcomes-but also when it rises significantly. For instance, an increase in RMSSD could represent pathological conditions, such as severe unconjugated hyperbilirubinemia (in association with low minimum HR) (Özdemir et al., 2018), or the occurrence of an antenatal/intrapartum hypoxia-ischemia that could lead to brain injury (Frasch, 2018; Yamaguchi et al., 2018).

To make HRV a gold standard, however, there is the need to deepen the knowledge about RMSSD or any other metric: more robust studies on larger samples are needed (Stéphan-Blanchard 
et al., 2013; De Jonckheere and Storme, 2019) as studies still show contradictory findings that merit careful analysis (Wang et al., 2016; Joshi et al., 2020).

A further crucial step is to integrate HRV signals with other vital signs, such as $\mathrm{SpO}_{2}$ and blood pressure, to improve its predictive power and reduce mortality (Sullivan et al., 2018; Kumar et al., 2020). Even further is the integration of information about the infant's gestation, labor, and perinatal period is essential: since NICU professionals have to deal with complex pathologies, including SIDS (Siren, 2017), technological tools that can elaborate all these types of information could become paramount to improve clinical care (Hemphill et al., 2011).

Therefore, in parallel with introducing a real-time HRV monitoring system, there might be the possibility to start using the tools already present in NICUs to collect a large amount of data about all the babies' vital signs (Kumar et al., 2020). This data could then be analyzed by ML algorithms, whose results the bedside monitors would display as one or more metrics, graphs, or models (Bravi et al., 2011; Hemphill et al., 2011; Seely et al., 2011).

Indeed, several fields in medicine share this need of collecting and using a large amount of data to improve patient care. An example is the neurocritical care where, as any other intensive care unit, "extreme complexity reigns": continuous multimodal monitoring is vital to guide an efficient decision-making process with the purpose of preventing complications and warranting survival, especially in case of emergency (Hemphill et al., 2011; Lara and Püttgen, 2018).

However, this means revolutionizing the NICU itself. First, the data must be stored and automatically controlled for artifacts. Then, all the medical devices have to communicate and to be synchronized with each other to assure the collected data are coherent; they could also connect to a central computer/hub that autonomously synchronizes the data process. Synchronization is essential: otherwise, data interpretation would become flawed. Lastly, clinicians could add laboratory values, imaging results, and medical documentation to the patient's record (Hemphill et al., 2011).

Advanced bioinformatics could then follow with the use of the many available techniques of data mining coming from statistics, ML, and AI. For example, ML or Deep Learning approaches can be used, including the ones based on decision trees and neural networks, especially dynamic Bayesian neural networks, which capture well non-linear phenomena with complex multimodal relationships. Based on the big-data of other patients, such approaches could elaborate the past and present information of the actual patient to predict the evolution of the clinical conditions. Moreover, they could visualize this prediction in more useful ways (e.g., from numerical probability to specific types of graphs) than raw data or simple statistical metrics (Hemphill et al., 2011).

Multimodal monitoring and the efficient application of data mining algorithms in medicine have yet to grow. In fact, many obstacles (e.g., the availability of resources) may hinder such development, but the complexity of intensive care units makes a strong call to action, and the stakeholders in the healthcare systems must be aware of the opportunities informatics could give to clinical care (Hemphill et al., 2011; Lara and Püttgen, 2018).

The stakeholders must also know that it is paramount to establish tight collaborations between the obstetric, neonatal, and pediatric departments: sharing data about patients' health and medical devices among these departments would elicit highquality research that would help clinicians to correlate neonatal outcomes to their fetal development and past history, thus truly improving neonatal care-otherwise, how would it be possible to treat neonatal pathologies ignoring their fetal origin?

In the next sections, it is argued as HRV analysis could indeed represent a useful tool to help both in decision-making and in monitoring the infant's condition.

\section{HRV as an Innovative Tool in Neonatal Decision-Making Intervention}

Because various metrics, such as RMSSD, relate to HRV modulation by vagal tone, which plays a significant role in infant's condition, HRV monitoring could give reliable information about the infant's level of stress (Mulkey and du Plessis, 2019).

Indeed, since LF and HF inversely correlated with salivary cortisol levels in both healthy and NICU admitted newborns (Hashiguchi et al., 2020), real-time HRV monitoring could assess the actual stress level of newborns. Moreover, since stress- and pain-related behaviors in newborns are difficult to discriminate, real-time HRV could represent a valuable tool to assess both neonatal stress and pain (Cremillieux et al., 2018; Zhang et al., 2019).

As neonatal stress and pain can adversely influence neurodevelopment and brain maturation, as well as future health and longevity (Holsti et al., 2006; Reynolds, 2013; Pillai Riddell et al., 2015; Simeoni et al., 2018), every procedure that can improve the newborn's condition is paramount, especially in case of prematurity or pathological conditions. Knowing when and how to apply such procedures is even more essential: a real-time HRV monitor that gives information about the actual newborn's level of stress could elicit NICU professionals to promptly adjust the NICU environment to improve the newborn's wellbeing and comfort.

Real-time HRV would help nurses to adopt correct behaviors for reducing visual and auditory overstimulation: in a complex environment, such as the NICU, it is indeed difficult to know exactly when to perform these procedures (Aita and Goulet, 2003).

Based on the value of the index shown by the HRV monitor, NICU staff could increase or decrease the temperature of the incubator, change the illumination intensity or type, and introduce positive stimuli: for instance, human voice or songs, even recorded, cloths with parent-scent, eye contact, and gentle touch (Ozawa et al., 2010; Stéphan-Blanchard et al., 2013; Weber and Harrison, 2019).

Real-time HRV could also help choose between "positive" procedures, such as facilitated tucking-manually supporting the baby by holding the upper and lower extremities in flexion-plus human voice or skin-to-skin contact between the mother and the newborn: these procedures, if well 
performed, should increase the level of newborn's comfort, and a HRV monitor could be able to show their actual benefit (Alexandre et al., 2013; Butruille et al., 2017; Marvin et al., 2019).

As shown in sections "The ANS Development and Its Relationship With HRV" and "Factors That Can Alter HRV Assessment", HRV metrics could change according to environmental factors. However, this knowledge must be deepened, and the faster way to achieve this result could be introducing HRV monitoring in as many NICUs as possible and by collecting a large amount of data, from which ML applications could extrapolate models or metrics suitable and clinically-sound for the above-mentioned conditions.

A real-time HRV monitor could help understand when newborns need analgesic medications to withstand better invasive and noxious procedures (Weber et al., 2019) or adjuvant neuroprotective therapies in case of HIEinfants who subsequently died due to HIE showed lower LF and higher HF-(Massaro et al., 2014), and it could also help choose better how to administer surfactant in case of respiratory distress syndrome-the NIPE index showed different values according to the technique used (Okur et al., 2019).

In the same way, HRV could guide the choice among various respiratory supports post-extubation, besides predicting the outcome of extubation. Indeed, RMSSD, SDNN, pNN50, $\mathrm{TP}$, and VLF were higher during non-synchronized noninvasive ventilation than during nasal continuous positive airway pressure (CPAP) in extreme preterms who were reintubated a few hours later (Latremouille et al., 2018). Such difference was not found in infants who showed successful extubation, even though every HRV metric analyzed was higher (but not significantly) during ventilation than nasal CPAP. Therefore, the authors speculated that those variations could correlate with the failure in extubation, and that noninvasive ventilation could better modulate the ANS of less stable infants than nasal CPAP (Latremouille et al., 2018). HRV measured as SDRR was also found to increase during high flow nasal cannula, but not during nasal CPAP, in extremely preterm infants who succeeded in being extubated (Latremouille et al., 2019).

\section{HRV as an Index to Monitor Clinical Improvement}

To be an all-round innovative tool, real-time HRV should not only predict future outcomes with good accuracy but should also provide useful information to monitor the current condition of the newborn.

The question is: could HRV help understand whether the baby's condition is deteriorating or improving?

A real-time HRV monitoring system could give a clear answer. Besides, this is another field in which more data and the use of ML software could dramatically increase the clinical usefulness of HRV, and thus another reason to introduce HRV monitors in NICU and to integrate their recordings with the infant's development over time.
HRV-measured through several metrics including HRC index, DFA $\alpha_{S}, \mathrm{RMS}_{S}, \mathrm{RMS}_{\mathrm{L}}, \mathrm{LF}$, and HF-could change greatly in case of pathological conditions, such as congenital heart disease, cardiac failure in persistent ductus arteriosus, respiratory deterioration with apnea, respiratory distress syndrome, inflammation, hyperbilirubinemia, respiratory syncytial virus, and sepsis. Indeed, inflammatory cytokines (e.g., IL-6, IL-8, and IL-13), bacterial toxins, and free bilirubin could pass through the immature brain-blood barrier and exert neurotoxic activity, thus negatively influencing the nervous system. The same negative effect can be induced by the cardiorespiratory pathologies (van Ravenswaaij-Arts et al., 1991; Prietsch et al., 1992; Stock et al., 2010; Raynor et al., 2012; Sullivan et al., 2014; Uhrikova et al., 2015; Al-Shargabi et al., 2017; Mulkey et al., 2020).

Since HRV modifications correlated with inflammatory cytokine levels (Al-Shargabi et al., 2017), real-time HRV could be a useful marker of clinical acuity: indeed, HRV alterations-a decrease in $\mathrm{RMS}_{S}, \mathrm{RMS}_{\mathrm{L}}$, DFA $\alpha_{S}, \mathrm{LF}, \mathrm{HF}$, and TP or an increase in HRC index-correlated with the pathology severity (Sullivan et al., 2014; Al-Shargabi et al., 2018). Variations in LF, HF, and LF/HF correlated with HIE severity, although more studies are needed to resolve conflicting results between studies (Andersen et al., 2019). In a recent paper on a pediatric population, compared with the time of admission in the intensive care units, RMSSD, SDNN, and pNN50 continuously rose as the time of discharge approached, that is, as the infants recovered (Marsillio et al., 2019).

The correlation between HRV alterations, inflammation, and inflammation-related pathologies can be better understood through the cholinergic anti-inflammatory pathway (CAP). Briefly, the CAP begins with the afferent vagus nerve that detects inflammatory cytokines or bacterial toxins and transmits these signals to several brain nuclei, which then induce an anti-inflammatory response through the release of acetylcholine. This response involves complex interactions between both ANS branches and plays a paramount role in finely tuning the immune response and avoiding tissue damages in case of inflammation (Garzoni et al., 2013; Bonaz et al., 2017).

The CAP can fail to properly function in case of ANS dysfunction, but also due to pathogens and inflammation: pathogens can rapidly activate but desensitize the CAP (i.e., alter cholinergic receptors) (Fairchild et al., 2011), whereas prolonged inflammation can induce apoptosis in the brainstem vagal nuclei (Fritze et al., 2014). As a result, HRV decreases, and adverse outcomes can occur: indeed, low vagal activity and reduced CAP efficiency correlate with increased inflammation, morbidity, and mortality, in both infants and adults (Garzoni et al., 2013; Bonaz et al., 2017).

In fetuses, the CAP, although at the early stage of maturation, might also modulate local and systemic inflammation (Garzoni et al., 2013). Indeed, animal studies suggested that in ovine fetuses, CIMVA applied to fHRV found several metrics that uniquely predicted, 1.5 days in advance, M1 and M2 macrophages activation and occludin expression (i.e., increased leakiness) in the terminal ileum (Liu et al., 2016). Therefore, 
if cytokines and toxins can alter HRV through the CAP, it might be intuitive why HRV analysis can inform about the global health of the infant and predict future inflammationrelated development.

HRV modifications could also reflect the effect of treatments, such as ventilation, antibiotics, and phototherapy. To date, a clear correlation is still lacking, and better research is needed, although initial evidence is available (Sullivan et al., 2014; Uhrikova et al., 2015; Al-Shargabi et al., 2017; Latremouille et al., 2018). For instance, as $\mathrm{RMS}_{\mathrm{S}}, \mathrm{RMS}_{\mathrm{L}}, \mathrm{DFA} \alpha_{\mathrm{S}}, \mathrm{LF}, \mathrm{HF}$, and TP decreased around $48 \mathrm{~h}$ before NEC occurred, the same metrics recovered to their baseline values during the first $60 \mathrm{~h}$ after NEC diagnosis (Al-Shargabi et al., 2018). Monitoring HRV in the $60 \mathrm{~min}$ prior to extubation could help predict which babies will have an adverse outcome, since they could show much lower TP, LF, HF, LF/HF, and VLF than babies who will succeed in being extubated (Kaczmarek et al., 2013).

Other indications about the clinical usefulness of HRV, and real-time monitoring, come from the studies on HIE severity and ANS in infants. During therapeutic hypothermia for HIE, lower $\mathrm{RMS}_{\mathrm{S}}, \mathrm{RMS}_{\mathrm{L}}, \mathrm{DFA} \alpha_{\mathrm{S}}$, and LF and higher HF indicated a higher risk of secondary energy failure, brain injury, and death, thus helping in identifying the infants who failed to respond to hypothermia and needed adjuvant neuroprotective therapies (Govindan et al., 2014; Massaro et al., 2014; Metzler et al., 2017). The analysis of DFA $\alpha_{S}, \mathrm{RMS}_{\mathrm{S}}$, and $\mathrm{RMS}_{\mathrm{L}}$ could also prove useful to discriminate among different types of brain injury, such as white matter injury, watershed stroke, basal ganglia injury, or global injury (Metzler et al., 2017). As shown with fHRV analysis, HRV monitoring, especially during labor, could also predict which newborns are at risk of developing neuroinflammation and, thus, brain injury-neuroinflammation is correlated to severe acidemia, and fHRV monitoring could detect it with $1 \mathrm{~h}$ of advance. Since therapeutic hypothermia is poorly applied, either because often the brain injury has already developed or because neonatologists lack tools to properly recognize which newborns require hypothermia, HRV monitoring could overcome this predicament (Frasch et al., 2014; Xu et al., 2014; Gold et al., 2019).

Real-time HRV is shown to be useful for monitoring how infants react to routine-care procedures, such as diaper changes or pupil examination, and for revealing neurological abnormalities. Indeed, infants with HIE and impaired ANSdefined as at least one alteration in LF, HF, DFA $\alpha_{S}, \mathrm{RMS}_{\mathrm{S}}$, or $\mathrm{RMS}_{\mathrm{L}}$ - showed HR, blood pressure, and cerebral blood flow alteration during those procedures. Moreover, the more the number of altered HRV metrics, the worse the outcome, i.e., moderate severe brain injury or death (Campbell et al., 2018).

Real-time HRV could also help mothers and fathers to monitor the interaction with the baby: using a feedback and feedforward process, parents could see how their actions affect their baby (Van Puyvelde et al., 2019a,b; these studies, however, examined how touch influenced RSA and RR intervals, not specific HRV metrics).

Furthermore, real-time HRV could help control how the infant's condition progresses in different positions (supine, prone, or side-lying). This monitoring would be useful, for example, to balance the need for proper oxygenation and avoiding adverse outcomes, such as SIDS. Infants who succumbed to SIDS showed lower RSA during all sleep stages (Kluge et al., 1988) and altered Poincaré plots compared with controls (Schechtman et al., 1992). Those infants showed also higher basal HR during QS (Kelly et al., 1986), lower HF, and higher LF before preapneic sighs than those infants who did not die from SIDS (Franco et al., 2003).

Newborns who develop SIDS seem to show an autonomic instability that impedes them from facing a life-threatening event (Elhaik, 2016; Zhao et al., 2016). Real-time HRV could help prevent this adverse scenario. Besides, due to the uncertainty still surrounding SIDS-for instance, infants who succumb to SIDS could even show the same RSA and HR, but higher LF than controls (Gordon et al., 1984)—, continuous monitoring data analyzed by AI algorithms could help shed more light on SIDS occurrence by giving useful information about the newborn's ANS condition.

Speaking of which, an ML algorithm based on boosted decision trees increased the predictive power of HRV during episodes of low mean blood pressure in preterm infants with a GA between 23 and 31 weeks. Although good results were obtained even with single metrics-among time, frequency, and non-linear ones, RMSSD gave the best area under the curve (0.87) - combining all the metrics through the mentioned algorithm gave an area under the curve of 0.97 for predicting short-term neurological outcomes (i.e., grade III/IV intraventricular hemorrhage or cystic periventricular leukomalacia, NEC, bronchopulmonary dysplasia, infection, and retinopathy) (Semenova et al., 2018).

Lastly, real-time HRV monitoring could give information about the neurological development. Indeed, newborn's HRV predicted the occurrence of neurological abnormalities-cerebral palsy, language or mental retardation, vision or hearing disability, or attention disorders - at 2 years (Thiriez et al., 2015; Dimitrijević et al., 2016). Twenty-four hour RMSSD, SDANN, and SDNN were particularly good predictors, respectively, 88.9, 83.3 , and $83.3 \%$ specificity and $100 \%$ sensibility using the threshold values of 17,38 , and $47 \mathrm{~ms}$-and also improved the prognostic value of general movements assessment: among newborns with poor repertoire general movements, those with higher RMSSD, SDANN, and SDNN showed better neurological development (Dimitrijević et al., 2016).

\section{HRV Potential Usefulness in Middle- and Low-Income Realities}

HRV could be a useful non-invasive tool to assess infant's conditions and to reduce the risk of adverse outcomes and, as the HeRO monitor showed, mortality from sepsis. This last result is worth noting since neonatal sepsis remains one of the major causes of neonatal mortality, together with prematurity and birth-related complications, and this holds especially in middleand low-income countries (WHO, 2016).

Indeed, only 15 in 48 Asian and African countries reported having an indicator about sepsis management in their Health Management Information Systems (WHO, 2017). In countries, 
such as South Africa and India, about 1 infant in 35 dies before the first birthday, even more than ten times compared with developed countries, such as Japan, Sweden, or Italy (about 1 in 350-500) (OECD, 2020). Closing this large gap by reducing worldwide neonatal mortality to less than 1 infant in 100 was deemed by the WHO as one of the top priorities in 2015 (WHO, 2016). Of notice, the first 28 days after birth shows the highest mortality risk (United Nations Inter-agency Group for Child Mortality Estimation (UN IGME), 2019).

The studies that investigated the reasons behind the difference in mortality highlighted that the barriers to efficient care were twofold. On the one hand, people tend to delay seeking care due to lack of knowledge about neonatal health, sociocultural behaviors (e.g., relying on traditional practices), and several concerns about the cost of healthcare, the attitude of NICU staff, and the lack of appropriate medical equipment (Watson et al., 2020). On the other hand, obstetrics and neonatal evidencebased practices fail to be introduced due to lack of financial resources, workforce capacity, and specific training in case of emergency. The effect is a low-quality screening for pathological conditions, such as IUGR or sepsis, which can lead to severe neonatal health problems and even death (Hoyer et al., 2017; Otieno et al., 2018).

To date, real-time HRV monitoring cannot solve all these complex problems, but it could surely address financial- and efficiency-related obstacles. Indeed, HRV monitors based on PPG, contactless vPPG, or even BSG could be low-cost and easyto-use devices (Sun et al., 2012; Sun and Thakor, 2016; Joshi et al., 2018) that allow midwifery and NICU staff to efficiently monitor the newborn's development, screen for pathological conditions, and choose the best treatment (Hoyer et al., 2017). A recent paper showed that the HeRO monitor could also help reduce, even if a modest effect was shown, the use of antibiotics and blood culture tests for suspicion of sepsis in the first 120 days of life (King, 2020).

Moreover, if relying on big-data and the Internet, the HRV monitors could even be simple systems that monitor the babies, send information to a central hub (e.g., through the Cloud), and receive the corresponding elaboration, thus having the

\section{REFERENCES}

Aarts, L. A. M., Jeanne, V., Cleary, J. P., Lieber, C., Nelson, J. S., Bambang Oetomo, S., et al. (2013). Non-contact heart rate monitoring utilizing camera photoplethysmography in the neonatal intensive care unit - A pilot study. Early Hum. Dev. 89, 943-948. doi: 10.1016/j.earlhumdev.2013.09.016

Abry, P., Roux, S. G., Chudacek, V., Borgnat, P., Goncalves, P., and Doret, M. (2013). "Hurst exponent and intrapartum fetal heart rate: impact of decelerations," in Proceedings of the 26th IEEE International Symposium on Computer-Based Medical Systems, Port: IEEE, 131-136. doi: 10.1109/CBMS. 2013.6627777

Aita, M., and Goulet, C. (2003). Assessment of neonatal nurses' behaviors that prevent overstimulation in preterm infants. Intensive Crit. Care Nurs. 19, 109-118. doi: 10.1016/s0964-3397(03) 00023-5

Aita, M., Johnston, C., Goulet, C., Oberlander, T. F., and Snider, L. (2013). Intervention minimizing preterm infants' exposure to NICU light and noise. Clin. Nurs. Res. 22, 337-358. doi: 10.1177/1054773812469223 same efficiency of HRV monitors in high-income countries (Werth, 2019).

\section{CONCLUSION}

The present paper reviewed the use of HRV in the neonatal field and the utility of real-time HRV monitoring to assess the newborn's clinical conditions, showing that several metrics and computed metrics change in conjunction with stress-/pain-related behaviors, inflammation, pathological conditions, such as cardiac failure, respiratory distress syndrome, hyperbilirubinemia, NEC, and sepsis, and neurological development.

The paper also reviewed the NICU technology to evaluate how to measure real-time HRV efficiently. Indeed, a system based on PPG could be the optimal solution due to being low-cost, easy-touse, and non-invasive, although PPG-based computation seems less precise than ECG-based computation. Therefore, future studies will have to carefully assess if the outcomes reviewed in this paper might be influenced by this difference in precision between PPG and ECG.

In the next decade, introducing real-time HRV in NICUs would be a great step forward in the improvement of neonatal care, especially if supported by the advancements in bioinformatics, which could easily extrapolate accurate predicting models from all the data collected in the NICUs, although several concerns and limitations have to be overcome before fully implementing the system into a daily NICU routine care.

\section{AUTHOR CONTRIBUTIONS}

MC, FCe, AC, NB, and AM contributed equally to the conceptualization and to the writing of the manuscript. DB, FCa, and $\mathrm{CC}$ revised and edited the final draft of the manuscript. All authors approved the final manuscript as submitted and agreed to be accountable for all aspects of the work.

Alexandre, C., De Jonckheere, J., Rakza, T., Mur, S., Carette, D., Logier, R., et al. (2013). [Impact of cocooning and maternal voice on the autonomic nervous system activity in the premature newborn infant]. Arch. Pediatr. 20, 963-968. doi: 10.1016/j.arcped.2013.06.006

Almadhoob, A., and Ohlsson, A. (2020). Sound reduction management in the neonatal intensive care unit for preterm or very low birth weight infants. Cochrane Database Syst. Rev. 1:CD010333. doi: 10.1002/14651858.CD010333. pub3

Alonzo, C. J., Nagraj, V. P., Zschaebitz, J. V., Lake, D. E., Moorman, J. R., and Spaeder, M. C. (2018). Heart rate ranges in premature neonates using high resolution physiologic data. J. Perinatol. 38, 1242-1245. doi: 10.1038/s41372018-0156-1

Als, H., Butler, S., Kosta, S., and McAnulty, G. (2005). The assessment of preterm infants' behavior (APIB): furthering the understanding and measurement of neurodevelopmental competence in preterm and full-term infants. Ment. Retard. Dev. Disabil. Res. Rev. 11, 94-102. doi: 10.1002/mrdd.20053

Al-Shargabi, T., Govindan, R. B., Dave, R., Metzler, M., Wang, Y., du Plessis, A., et al. (2017). Inflammatory cytokine response and reduced heart rate variability 
in newborns with hypoxic-ischemic encephalopathy. J. Perinatol. 37, 668-672. doi: $10.1038 /$ jp.2017.15

Al-Shargabi, T., Reich, D., Govindan, R. B., Shankar, S., Metzler, M., Cristante, C., et al. (2018). Changes in autonomic tone in premature infants developing necrotizing enterocolitis. Am. J. Perinatol. 35, 1079-1086. doi: 10.1055/s-00381639339

Andersen, M., Andelius, T. C. K., Pedersen, M. V., Kyng, K. J., and Henriksen, T. B. (2019). Severity of hypoxic ischemic encephalopathy and heart rate variability in neonates: a systematic review. BMC Pediatr. 19:242. doi: 10.1186/s12887019-1603-7

Aye, C. Y. L., Lewandowski, A. J., Oster, J., Upton, R., Davis, E., Kenworthy, Y., et al. (2018). Neonatal autonomic function after pregnancy complications and early cardiovascular development. Pediatr. Res. 84, 85-91. doi: 10.1038/s41390018-0021-0

Ayres-de-Campos, D., and Bernardes, J. (2010). Twenty-five years after the FIGO guidelines for the use of fetal monitoring: time for a simplified approach? Int. J. Gynecol. Obstet. 110, 1-6. doi: 10.1016/j.ijgo.2010.03.011

Babenko, O., Kovalchuk, I., and Metz, G. A. (2015). Stress-induced perinatal and transgenerational epigenetic programming of brain development and mental health. Neurosci. Biobehav. Rev. 48, 70-91. doi: 10.1016/j.neubiorev.2014.11. 013

Bellieni, C. V., Acampa, M., Maffei, M., Maffei, S., Perrone, S., Pinto, I., et al. (2008). Electromagnetic fields produced by incubators influence heart rate variability in newborns. Arch. Dis. Child Fetal Neonatal Ed. 93, F298-F301. doi: 10.1136/adc.2007.132738

Benarroch, E. E. (1993). The central autonomic network: functional organization, dysfunction, and perspective. Mayo Clin. Proc. 68, 988-1001. doi: 10.1016/ s0025-6196(12)62272-1

Béres, S., Holczer, L., and Hejjel, L. (2019). On the minimal adequate sampling frequency of the photoplethysmogram for pulse rate monitoring and heart rate variability analysis in mobile and wearable technology. Meas. Sci. Rev. 19, 232-240. doi: 10.2478/msr-2019-0030

Biban, P. (2010). From neonatal to paediatric intensive care: an educational pathway. Minerva Pediatr. 62(3 Suppl. 1), 129-131.

Billman, G. E. (2011). Heart rate variability - a historical perspective. Front. Physiol. 2:86. doi: $10.3389 /$ fphys.2011.00086

Blackford, E. B., Piasecki, A. M., and Estepp, J. R. (2016). Measuring pulse rate variability using long-range, non-contact imaging photoplethysmography. Conf. Proc. IEEE Eng. Med. Biol. Soc. 2016, 3930-3936. doi: 10.1109/EMBC. 2016.7591587

Blanik, N., Abbas, A. K., Venema, B., Blazek, V., and Leonhardt, S. (2014). Hybrid optical imaging technology for long-term remote monitoring of skin perfusion and temperature behavior. J. Biomed. Opt. 19:016012. doi: 10.1117/1.JBO.19.1. 016012

Blanik, N., Heimann, K., Pereira, C., Paul, M., Blazek, V., Venema, B., et al. (2016). Remote vital parameter monitoring in neonatology - robust, unobtrusive heart rate detection in a realistic clinical scenario. Biomed. Tech. 61, 631-643. doi: 10.1515/bmt-2016-0025

Bonaz, B., Sinniger, V., and Pellissier, S. (2017). The vagus nerve in the neuroimmune axis: implications in the pathology of the gastrointestinal tract. Front. Immunol. 8:1452. doi: 10.3389/fimmu.2017.01452

Bravi, A., Longtin, A., and Seely, A. J. (2011). Review and classification of variability analysis techniques with clinical applications. Biomed. Eng. Online 10:90. doi: 10.1186/1475-925X-10-90

Butruille, L., Blouin, A., De Jonckheere, J., Mur, S., Margez, T., Rakza, T., et al. (2017). Impact of skin-to-skin contact on the autonomic nervous system in the preterm infant and his mother. Infant Behav. Dev. 49, 83-86. doi: 10.1016/j. infbeh.2017.07.003

Butruille, L., De Jonckheere, J., Marcilly, R., Boog, C., Bras da Costa, S., Rakza, T., et al. (2015). Development of a pain monitoring device focused on newborn infant applications: the NeoDoloris project. IRBM 36, 80-85. doi: 10.1016/j. irbm.2015.01.005

Buyuktiryaki, M., Uras, N., Okur, N., Oncel, M. Y., Simsek, G. K., Isik, S. O., et al. (2018). Evaluation of prolonged pain in preterm infants with pneumothorax using heart rate variability analysis and EDIN (Échelle Douleur Inconfort Nouveau-Né, neonatal pain and discomfort scale) scores. Korean J. Pediatr. 61, 322-326. doi: $10.3345 / \mathrm{kjp} .2017 .05939$
Campbell, H., Govindan, R. B., Kota, S., Al-Shargabi, T., Metzler, M., Andescavage, N., et al. (2018). Autonomic dysfunction in neonates with hypoxic ischemic encephalopathy undergoing therapeutic hypothermia impairs physiological responses to routine care events. J. Pediatr. 196, 38-44. doi: 10.1016/j.jpeds. 2017.12.071

Cardoso, S., Silva, M. J., and Guimarães, H. (2017). Autonomic nervous system in newborns: a review based on heart rate variability. Childs. Nerv. Syst. 33, 1053-1063. doi: 10.1007/s00381-017-3436-8

Cesarelli, M., Romano, M., Ruffo, M., Bifulco, P., and Pasquariello, G. (2010). Foetal heart rate variability frequency characteristics with respect to uterine contractions. J. Biomed. Sci. Eng. 03, 1014-1021. doi: 10.4236/jbise.2010. 310132

Cheng, G., Zhou, X., Qu, J., Ashwell, K. W., and Paxinos, G. (2004). Central vagal sensory and motor connections: human embryonic and fetal development. Auton. Neurosci. 114, 83-96. doi: 10.1016/j.autneu.2004.06.008

Chiew, C. J., Liu, N., Tagami, T., Wong, T. H., Koh, Z. X., and Ong, M. E. H. (2019). Heart rate variability based machine learning models for risk prediction of suspected sepsis patients in the emergency department. Medicine 98:e14197. doi: 10.1097/MD.00000000 00014197

Chock, V. Y., Davis, A. S., and Hintz, S. R. (2015). the roles and responsibilities of the neonatologist in complex fetal medicine: providing a continuum of care. Neoreviews 16, e9-e15. doi: 10.1542/neo.16-1-e9

Choi, A., and Shin, H. (2017). Photoplethysmography sampling frequency: pilot assessment of how low can we go to analyze pulse rate variability with reliability? Physiol. Meas. 38, 586-600. doi: 10.1088/1361-6579/aa5efa

Chudacek, V., Anden, J., Mallat, S., Abry, P., and Doret, M. (2014). Scattering transform for intrapartum fetal heart rate variability fractal analysis: a casecontrol study. IEEE Trans. Biomed. Eng. 61, 1100-1108. doi: 10.1109/TBME. 2013.2294324

Clairambault, J., Curzi-Dascalova, L., Kauffmann, F., Médigue, C., and Leffler, C. (1992). Heart rate variability in normal sleeping full-term and preterm neonates. Early Hum. Dev. 28, 169-183. doi: 10.1016/0378-3782(92)90 $111-\mathrm{s}$

Cobos-Torres, J. C., Abderrahim, M., and Martínez-Orgado, J. (2018). Noncontact, simple neonatal monitoring by photoplethysmography. Sensors 18:4362. doi: 10.3390/s18124362

Cremillieux, C., Makhlouf, A., Pichot, V., Trombert, B., and Patural, H. (2018). Objective assessment of induced acute pain in neonatology with the Newborn Infant Parasympathetic Evaluation index. Eur. J. Pain. 22, 1071-1079. doi: 10. 1002/ejp.1191

Cunningham, S., Symon, A. G., Elton, R. A., Zhu, C., and McIntosh, N. (1999). Intra-arterial blood pressure reference ranges, death and morbidity in very low birthweight infants during the first seven days of life. Early Hum. Dev. 56, 151-165. doi: 10.1016/s0378-3782(99)00038-9

Daunhawer, I., Kasser, S., Koch, G., Sieber, L., Cakal, H., Tütsch, J., et al. (2019). Enhanced early prediction of clinically relevant neonatal hyperbilirubinemia with machine learning. Pediatr. Res. 86, 122-127. doi: 10.1038/s41390-0190384-x

De Jonckheere, J., and Storme, L. (2019). NIPE is related to parasympathetic activity. Is it also related to comfort? J. Clin. Monit. Comput. 33, 747-748. doi: 10.1007/s10877-019-00276-1

de Souza Filho, L. F. M., de Oliveira, J. C. M., Ribeiro, M. K. A., Moura, M. C., Fernandes, N. D., de Sousa, R. D., et al. (2019). Evaluation of the autonomic nervous system by analysis of heart rate variability in the preterm infants. BMC Cardiovasc. Disord. 19:198. doi: 10.1186/s12872-01 9-1166-4

DiPietro, J. A., and Voegtline, K. M. (2017). The gestational foundation of sex differences in development and vulnerability. Neuroscience 342, 4-20. doi: 10 . 1016/j.neuroscience.2015.07.068

Dereymaeker, A., Pillay, K., Vervisch, J., De Vos, M., Van Huffel, S., Jansen, K., et al. (2017). Review of sleep-EEG in preterm and term neonates. Early Hum. Dev. 113, 87-103. doi: 10.1016/j.earlhumdev.2017.07.003

Dimitrijević, L., Bjelaković, B., Čolović, H., Mikov, A., Živković, V., Kocić, M., et al. (2016). Assessment of general movements and heart rate variability in prediction of neurodevelopmental outcome in preterm infants. Early Hum. Dev. 99, 7-12. doi: 10.1016/j.earlhumdev.2016.05.014 
DiPietro, J. A., Hodgson, D. M., Costigan, K. A., Hilton, S. C., and Johnson, T. R. (1996). Fetal neurobehavioral development. Child. Dev. 67, 2553-2567. doi: 10.1111/j.1467-8624.1996.tb01874.x

Doheny, K. K., Palmer, C., Browning, K. N., Jairath, P., Liao, D., He, F., et al. (2014). Diminished vagal tone is a predictive biomarker of necrotizing enterocolitisrisk in preterm infants. Neurogastroenterol. Motil. 26, 832-840. doi: 10.1111/ nmo. 12337

Durosier, L. D., Herry, C. L., Cortes, M., Cao, M., Burns, P., Desrochers, A., et al. (2015). Does heart rate variability reflect the systemic inflammatory response in a fetal sheep model of lipopolysaccharide-induced sepsis? Physiol. Meas. 36, 2089-2102. doi: 10.1088/0967-3334/36/10/2089

Eiselt, M., Curzi-Dascalova, L., Clairambault, J., Kauffmann, F., Médigue, C., and Peirano, P. (1993). Heart-rate variability in low-risk prematurely born infants reaching normal term: a comparison with full-term newborns. Early Hum. Dev. 32, 183-195. doi: 10.1016/0378-3782(93)90011-I

Elgendi, M., Norton, I., Brearley, M., Dokos, S., Abbott, D., and Schuurmans, D. (2016). A pilot study: can heart rate variability (HRV) be determined using short-term photoplethysmograms? F1000Res. 5:2354. doi: 10.12688/ f1000research.9556.1

Elhaik, E. (2016). A "Wear and Tear" hypothesis to explain sudden infant death syndrome. Front. Neurol. 7:180. doi: 10.3389/fneur.2016.00180

Fairchild, K., and Aschner, J. (2012). HeRO monitoring to reduce mortality in NICU patients. Res. Rep. Neonatol. 2, 65-76. doi: 10.2147/RRN.S32570

Fairchild, K. D. (2013). Predictive monitoring for early detection of sepsis in neonatal ICU patients. Curr. Opin. Pediatr. 25, 172-179. doi: 10.1097/MOP. 0b013e32835e8fe6

Fairchild, K. D., and O'Shea, T. M. (2010). Heart rate characteristics: physiomarkers for detection of late-onset neonatal sepsis. Clin. Perinatol. 37, 581-598. doi: 10.1016/j.clp.2010.06.002

Fairchild, K. D., Srinivasan, V., Randall Moorman, J., Gaykema, R. P. A., and Goehler, L. E. (2011). Pathogen-induced heart rate changes associated with cholinergic nervous system activation. Am. J. Physiol. Regul. Integr. Comp. Physiol. 300, R330-R339. doi: 10.1152/ajpregu.00487.2010

Ferrario, M., Signorini, M. G., Magenes, G., and Cerutti, S. (2006). Comparison of entropy-based regularity estimators: application to the fetal heart rate signal for the identification of fetal distress. IEEE Trans. Biomed. Eng. 53, 119-125. doi: 10.1109/TBME.2005.859809

Forte, G., and Casagrande, M. (2019). Heart rate variability and cognitive function: a systematic review. Front. Neurosci. 13:710. doi: 10.3389/fnins.2019.00710

Fox, G. P., and Matthews, T. G. (1989). Autonomic dysfunction at different ambient temperatures in infants at risk of sudden infant death syndrome. Lancet 2, 1065-1067. doi: 10.1016/s0140-6736(89)91080-5

Franco, P., Szliwowski, H., Dramaix, M., and Kahn, A. (2000). Influence of ambient temperature on sleep characteristics and autonomic nervous control in healthy infants. Sleep 23, 401-407.

Franco, P., Verheulpen, D., Valente, F., Kelmanson, I., de Broca, A., Scaillet, S., et al. (2003). Autonomic responses to sighs in healthy infants and in victims of sudden infant death. Sleep Med. 4, 569-577. doi: 10.1016/s1389-9457(03) 00107-2

Frasch, M. G. (2018). Saving the brain one heartbeat at a time: perspectives. J. Physiol. 596, 5503-5504. doi: 10.1113/JP275776

Frasch, M. G. (2020). Heart rate variability code: does it exist and can we hack it? ArXiv [Preprint], Available online at: http://arxiv.org/abs/2001.08264 (accessed July 25, 2020).

Frasch, M. G., Herry, C. L., Niu, Y., and Giussani, D. A. (2020). First evidence that intrinsic fetal heart rate variability exists and is affected by hypoxic pregnancy. J. Physiol. 598, 249-263. doi: 10.1113/JP278773

Frasch, M. G., Szynkaruk, M., Prout, A. P., Nygard, K., Cao, M., Veldhuizen, R., et al. (2016). Decreased neuroinflammation correlates to higher vagus nerve activity fluctuations in near-term ovine fetuses: a case for the afferent cholinergic anti-inflammatory pathway? J. Neuroinflammation 13:103. doi: 10. 1186/s12974-016-0567-x

Frasch, M. G., Xu, Y., Stampalija, T., Durosier, L. D., Herry, C., Wang, X., et al. (2014). Correlating multidimensional fetal heart rate variability analysis with acid-base balance at birth. Physiol. Meas. 35, L1-L12. doi: 10.1088/0967-3334/ 35/12/L1

Frasch, M. G., Zwiener, U., Hoyer, D., and Eiselt, M. (2007). Autonomic organization of respirocardial function in healthy human neonates in quiet and active sleep. Early Hum. Dev. 83, 269-277. doi: 10.1016/j.earlhumdev.2006.05. 023

Fraser, A. G. (2017). A manifesto for cardiovascular imaging: addressing the human factor. Eur. Heart J. Cardiovasc. Imaging 18, 1311-1321. doi: 10.1093/ehjci/ jex216

Fritze, D., Zhang, W., Li, J.-Y., Chai, B., and Mulholland, M. (2014). Thrombin mediates vagal apoptosis and dysfunction in inflammatory bowel disease. J. Gastrointest. Surg. 18, 1495-1506. doi: 10.1007/s11605-014-2565-6

Fyfe, K. L., Yiallourou, S. R., Wong, F. Y., Odoi, A., Walker, A. M., and Horne, R. S. C. (2015). The effect of gestational age at birth on post-term maturation of heart rate variability. Sleep 38, 1635-1644. doi: 10.5665/sleep.5064

Galland, B. C., Taylor, B. J., Bolton, D. P., and Sayers, R. M. (2006). Heart rate variability and cardiac reflexes in small for gestational age infants. J. Appl. Physiol. 100, 933-939. doi: 10.1152/japplphysiol.01275.2005

Gardner, F. C., Adkins, C. S., Hart, S. E., Travagli, R. A., and Doheny, K. K. (2018). Preterm stress behaviors, autonomic indices, and maternal perceptions of infant colic. Adv. Neonatal. Care 18, 49-57. doi: 10.1097/ANC.0000000000000451

Garzoni, L., Faure, C., and Frasch, M. G. (2013). Fetal cholinergic antiinflammatory pathway and necrotizing enterocolitis: the brain-gut connection begins in utero. Front. Integr. Neurosci. 7:57. doi: 10.3389/fnint.2013.00057

Georgieva, A., Payne, S. J., Moulden, M., and Redman, C. W. G. (2013). Artificial neural networks applied to fetal monitoring in labour. Neural Comput. Appl. 22, 85-93. doi: 10.1007/s00521-011-0743-y

Gold, N., Herry, C. L., Wang, X., and Frasch, M. G. (2019). Fetal cardiovascular decompensation during labor predicted from the individual heart rate: a prospective study in fetal sheep near term and the impact of low sampling rate. ArXiv [Preprint], Available online at: http://arxiv.org/abs/1911.01304 (accessed August 21, 2020).

Gonçalves, H., Amorim-Costa, C., Ayres-de-Campos, D., and Bernardes, J. (2017). Gender-specific evolution of fetal heart rate variability throughout gestation: a study of 8823 cases. Early Hum. Dev. 115, 38-45. doi: 10.1016/j.earlhumdev. 2017.09.002

Gonçalves, H., Rocha, A. P., Ayres-de-Campos, D., and Bernardes, J. (2006). Linear and nonlinear fetal heart rate analysis of normal and acidemic fetuses in the minutes preceding delivery. Med. Biol. Eng. Comput. 44, 847-855. doi: 10.1007/ s11517-006-0105-6

Gordon, D., Cohen, R. J., Kelly, D., Akselrod, S., and Shannon, D. C. (1984). Sudden infant death syndrome: abnormalities in short term fluctuations in heart rate and respiratory activity. Pediatr. Res. 18, 921-926. doi: 10.1203/ 00006450-198410000-00001

Govindan, R. B., Massaro, A. N., Al-Shargabi, T., Andescavage, N. N., Chang, T., Glass, P., et al. (2014). Detrended fluctuation analysis of non-stationary cardiac beat-to-beat interval of sick infants. EPL Europhys. Lett. 108:40005. doi: 10.1209/0295-5075/108/40005

Griffin, M. P., Lake, D. E., Bissonette, E. A., Harrell, F. E. Jr., O’Shea, T. M., and Moorman, J. R. (2005). Heart rate characteristics: novel physiomarkers to predict neonatal infection and death. Pediatrics 116, 1070-1074. doi: 10.1542/ peds.2004-2461

Griffin, M. P., and Moorman, J. R. (2001). Toward the early diagnosis of neonatal sepsis and sepsis-like illness using novel heart rate analysis. Pediatrics 107, 97-104. doi: 10.1542/peds.107.1.97

Hashiguchi, K., Kuriyama, N., Koyama, T., Matsui, D., Ozaki, E., Hasegawa, T., et al. (2020). Validity of stress assessment using heart rate variability in newborns. Pediatr. Int. 62, 694-700. doi: 10.1111/ped.14149

Hay, W. W. (1987). The uses, benefits, and limitations of pulse oximetry in neonatal medicine: consensus on key issues. J. Perinatol. 7, 347-349.

Hayano, J., and Yuda, E. (2019). Pitfalls of assessment of autonomic function by heart rate variability. J. Physiol. Anthropol. 38:3. doi: 10.1186/s40101-01 9-0193-2

Heathers, J. A. (2012). Sympathovagal balance from heart rate variability: an obituary. Exp. Physiol. 97:556. doi: 10.1113/expphysiol.2011.063867

Hejjel, L. (2017). Comment on 'Photoplethysmography sampling frequency: pilot assessment of how low can we go to analyze pulse rate variability with reliability?'. Physiol. Meas. 38, 2249-2251. doi: 10.1088/1361-6579/aa 9303

Hemphill, J. C., Andrews, P., and De Georgia, M. (2011). Multimodal monitoring and neurocritical care bioinformatics. Nat. Rev. Neurol. 7, 451-460. doi: 10. 1038/nrneurol.2011.101 
Henry, C., Shipley, L., Ward, C., Mirahmadi, S., Liu, C., Morgan, S., et al. (2020). Accurate neonatal heart rate monitoring using a new wireless, cap mounted device. Acta Paediatr. doi: 10.1111/apa.15303 [Epub ahead of print].

Herry, C. L., Burns, P., Desrochers, A., Fecteau, G., Durosier, L. D., Cao, M., et al. (2019). Vagal contributions to fetal heart rate variability: an omics approach. Physiol. Meas. 40:065004. doi: 10.1088/1361-6579/ab21ae

Herry, C. L., Cortes, M., Wu, H.-T., Durosier, L. D., Cao, M., Burns, P., et al. (2016). Temporal patterns in sheep fetal heart rate variability correlate to systemic cytokine inflammatory response: a methodological exploration of monitoring potential using complex signals bioinformatics. PLoS One 11:e0153515. doi: 10.1371/journal.pone.0153515

Holsti, L., Grunau, R. E., Whifield, M. F., Oberlander, T. F., and Lindh, V. (2006). Behavioral responses to pain are heightened after clustered care in preterm infants born between 30 and 32 weeks gestational age. Clin. J. Pain 22, 757-764. doi: 10.1097/01.ajp.0000210921.10912.47

Holzman, J. B., and Bridgett, D. J. (2017). Heart rate variability indices as biomarkers of top-down self-regulatory mechanisms: a meta-analytic review. Neurosci. Biobehav. Rev. 74(Pt A), 233-255. doi: 10.1016/j.neubiorev.2016

Hoyer, D., Heinicke, E., Jaekel, S., Tetschke, F., Di Pietro Paolo, D., Haueisen, J., et al. (2009). Indices of fetal development derived from heart rate patterns. Early Hum. Dev. 85, 379-386. doi: 10.1016/j.earlhumdev.2009.01.002

Hoyer, D., Schmidt, A., Gustafson, K. M., Lobmaier, S. M., Lakhno, I., van Leeuwen, P., et al. (2019). Heart rate variability categories of fluctuation amplitude and complexity: diagnostic markers of fetal development and its disturbances. Physiol. Meas. 40:064002. doi: 10.1088/1361-6579/ab205f

Hoyer, D., Schneider, U., Kowalski, E.-M., Schmidt, A., Witte, O. W., Schleußner, E., et al. (2015). Validation of functional fetal autonomic brain age score fABAS in 5 min short recordings. Physiol. Meas. 36, 2369-2378. doi: 10.1088/09673334/36/11/2369

Hoyer, D., Tetschke, F., Jaekel, S., Nowack, S., Witte, O. W., Schleußner, E., et al. (2013). Fetal functional brain age assessed from universal developmental indices obtained from neuro-vegetative activity patterns. PLoS One 8:e74431. doi: 10.1371/journal.pone.0074431

Hoyer, D., Żebrowski, J., Cysarz, D., Gonçalves, H., Pytlik, A., Amorim-Costa, C., et al. (2017). Monitoring fetal maturation-objectives, techniques and indices of autonomic function. Physiol. Meas. 38, R61-R88. doi: 10.1088/1361-6579/ aa5fca

Jarczok, M. N., Kleber, M. E., Koenig, J., Loerbroks, A., Herr, R. M., Hoffmann, K., et al. (2015). Investigating the associations of self-rated health: heart rate variability is more strongly associated than inflammatory and other frequently used biomarkers in a cross sectional occupational sample. PLoS One 10:e0117196. doi: 10.1371/journal.pone.0117196

Javorka, K., Lehotska, Z., Kozar, M., Uhrikova, Z., Kolarovszki, B., Javorka, M., et al. (2017). Heart rate variability in newborns. Physiol. Res. 66(Suppl. 2), S203-S214. doi: 10.33549/physiolres.933676

Jean-Louis, M., Anwar, M., Rosen, H., Craelius, W., Hiatt, M., and Hegyi, T. (2004). Power spectral analysis of heart rate in relation to sleep position. Biol. Neonate 86, 81-84. doi: 10.1159/000077782

Jeng, S. F., Yau, K. I., Chen, L. C., and Hsiao, S. F. (2000). Alberta infant motor scale: reliability and validity when used on preterm infants in Taiwan. Phys. Ther. 80, 168-178. doi: 10.1093/ptj/80.2.168

Jennings, J. R., Allen, B., Gianaros, P. J., Thayer, J. F., and Manuck, S. B. (2015). Focusing neurovisceral integration: cognition, heart rate variability, and cerebral blood flow. Psychophysiology 52, 214-224. doi: 10.1111/psyp.12319

Joshi, R., Bierling, B., Feijs, L., van Pul, C., and Andriessen, P. (2019a). Monitoring the respiratory rate of preterm infants using an ultrathin film sensor embedded in the bedding: a comparative feasibility study. Physiol. Meas. 40:045003. doi: 10.1088/1361-6579/ab1595

Joshi, R., Kommers, D., Guo, C., Bikker, J.-W., Feijs, L., van Pul, C., et al. (2019b). Statistical modeling of heart rate variability to unravel the factors affecting autonomic regulation in preterm infants. Sci. Rep. 9:7691. doi: 10.1038/s41598019-44209-z

Joshi, R., Bierling, B. L., Long, X., Weijers, J., Feijs, L., Van Pul, C., et al. (2018). A ballistographic approach for continuous and non-obtrusive monitoring of movement in neonates. IEEE J. Transl. Eng. Health Med. 6, 1-10. doi: 10.1109/ JTEHM.2018.2875703

Joshi, R., Kommers, D., Oosterwijk, L., Feijs, L., van Pul, C., and Andriessen, P. (2020). Predicting neonatal sepsis using features of heart rate variability, respiratory characteristics, and ECG-Derived estimates of infant motion. IEEE J. Biomed. Health Inform. 24, 681-692. doi: 10.1109/JBHI.2019. 2927463

Kaczmarek, J., Chawla, S., Marchica, C., Dwaihy, M., Grundy, L., and Sant'Anna, G. M. (2013). Heart rate variability and extubation readiness in extremely preterm infants. Neonatology 104, 42-48. doi: 10.1159/000347101

Kasai, M., Lear, C. A., Davidson, J. O., Beacom, M. J., Drury, P. P., Maeda, Y., et al. (2019). Early sinusoidal heart rate patterns and heart rate variability to assess hypoxia-ischaemia in near-term fetal sheep. J. Physiol. 597, 5535-5548. doi: 10.1113/JP278523

Kelly, D. H., Golub, H., Carley, D., and Shannon, D. C. (1986). Pneumograms in infants who subsequently died of sudden infant death syndrome. J. Pediatr. 109, 249-254. doi: 10.1016/s0022-3476(86)80380-8

Kevat, A. C., Bullen, D. V., Davis, P. G., and Kamlin, C. O. (2017). A systematic review of novel technology for monitoring infant and newborn heart rate. Acta Paediatr. 106, 710-720. doi: 10.1111/apa.13786

Kim, H. G., Cheon, E. J., Bai, D. S., Lee, Y. H., and Koo, B. H. (2018). Stress and heart rate variability: a meta-analysis and review of the literature. Psychiatry Investig. 15, 235-245. doi: 10.30773/pi.2017.08.17

King, W. E. (2020). HeRO monitoring: does it lead to unnecessary testing and treatment? Neonatology Today 15, 33-38.

Kluge, K. A., Harper, R. M., Schechtman, V. L., Wilson, A. J., Hoffman, H. J., and Southall, D. P. (1988). Spectral analysis assessment of respiratory sinus arrhythmia in normal infants and infants who subsequently died of sudden infant death syndrome. Pediatr. Res. 24, 677-682. doi: 10.1203/00006450198812000-00005

Koutcherov, Y., Mai, J. K., and Paxinos, G. (2003). Hypothalamus of the human fetus. J. Chem. Neuroanat. 26, 253-270. doi: 10.1016/j.jchemneu.2003.07.002

Kumar, N., Akangire, G., Sullivan, B., Fairchild, K., and Sampath, V. (2020). Continuous vital sign analysis for predicting and preventing neonatal diseases in the twenty-first century: big data to the forefront. Pediatr. Res. 87, 210-220. doi: 10.1038/s41390-019-0527-0

Laborde, S., Mosley, E., and Thayer, J. F. (2017). Heart rate variability and cardiac vagal tone in psychophysiological research - recommendations for experiment planning, data analysis, and data reporting. Front. Psychol. 8:213. doi: 10.3389/ fpsyg.2017.00213

Lakhno, I. (2017). Autonomic imbalance captures maternal and fetal circulatory response to pre-eclampsia. Clin. Hypertens 23:5. doi: 10.1186/s40885-0160061-x

Lara, L. R., and Püttgen, H. A. (2018). Multimodality monitoring in the neurocritical care unit: contin. Lifelong Learn. Neurol. 24, 1776-1788. doi: 10. 1212/CON.0000000000000671

Latremouille, S., Al-Jabri, A., Lamer, P., Kanbar, L., Shalish, W., Kearney, R. E., et al (2018). Heart rate variability in extremely preterm infants receiving nasal CPAP and non-synchronized noninvasive ventilation immediately after extubation. Respir. Care 63, 62-69. doi: 10.4187/respcare.05672

Latremouille, S., Shalish, W., Kanbar, L., Lamer, P., Rao, S., Kearney, R. E., et al. (2019). The effects of nasal continuous positive airway pressure and high flow nasal cannula on heart rate variability in extremely preterm infants after extubation: a randomized crossover trial. Pediatr. Pulmonol. 54, 788-796. doi: 10.1002/ppul.24284

Lee, W., Yoon, H., Han, C., Joo, K., and Park, K. (2016). Physiological signal monitoring bed for infants based on load-cell sensors. Sensors 16:409. doi: $10.3390 /$ s16030409

Li, D. K., Ferber, J. R., Odouli, R., and Quesenberry, C. P. Jr. (2012). A prospective study of in-utero exposure to magnetic fields and the risk of childhood obesity. Sci. Rep. 2:540. doi: 10.1038/srep00540

Li, X., Xu, Y., Herry, C., Durosier, L. D., Casati, D., Stampalija, T., et al. (2015). Sampling frequency of fetal heart rate impacts the ability to predict $\mathrm{pH}$ and BE at birth: a retrospective multi-cohort study. Physiol. Meas. 36, L1-L12. doi: 10.1088/0967-3334/36/5/L1

Li, X., Zheng, D., Zhou, S., Tang, D., Wang, C., and Wu, G. (2005). Approximate entropy of fetal heart rate variability as a predictor of fetal distress in women at term pregnancy. Acta Obstet. Gynecol. Scand. 84, 837-843. doi: 10.1111/j.00016349.2005.00773.x

Lin, T., Khalpey, Z., and Aras, S. (2020). Heart rate variability: a possible machine learning biomarker for mechanical circulatory device complications and heart recovery. VAD J. 6. [Epub ahead of print] 
Liu, H. L., Garzoni, L., Herry, C., Durosier, L. D., Cao, M., Burns, P., et al. (2016). Can monitoring fetal intestinal inflammation using heart rate variability analysis signal incipient necrotizing enterocolitis of the neonate?: pediatr. Crit. Care Med. 17, e165-e176. doi: 10.1097/PCC.0000000000000643

Lobmaier, S. M., Müller, A., Zelgert, C., Shen, C., Su, P. C., Schmidt, G., et al. (2020). Fetal heart rate variability responsiveness to maternal stress, noninvasively detected from maternal transabdominal ECG. Arch. Gynecol. Obstet. 301, 405-414. doi: 10.1007/s00404-019-05390-8

Lucchini, M., Fifer, W. P., Sahni, R., and Signorini, M. G. (2016). Novel heart rate parameters for the assessment of autonomic nervous system function in premature infants. Physiol. Meas. 37, 1436-1446. doi: 10.1088/0967-3334/37/9/ 1436

Malacova, E., Tippaya, S., Bailey, H. D., Chai, K., Farrant, B. M., Gebremedhin, A. T., et al. (2020). Stillbirth risk prediction using machine learning for a large cohort of births from Western Australia, 1980-2015. Sci. Rep. 10:5354. doi: 10.1038/s41598-020-62210-9

Marsillio, L. E., Manghi, T., Carroll, M. S., Balmert, L. C., and Wainwright, M. S. (2019). Heart rate variability as a marker of recovery from critical illness in children. PLoS One 14:e0215930. doi: 10.1371/journal.pone.0215930

Marvin, M. M., Gardner, F. C., Sarsfield, K. M., Travagli, R. A., and Doheny, K. K. (2019). Increased frequency of skin-to-skin contact is associated with enhanced vagal tone and improved health outcomes in preterm neonates. Am. J. Perinatol. 36, 505-510. doi: 10.1055/s-0038-1669946

Massaro, A. N., Campbell, H. E., Metzler, M., Al-Shargabi, T., Wang, Y., du Plessis, A., et al. (2017). Effect of temperature on heart rate variability in neonatal ICU Patients with hypoxic-ischemic encephalopathy. Pediatr. Crit. Care Med. 18, 349-354. doi: 10.1097/PCC.0000000000001094

Massaro, A. N., Govindan, R. B., Al-Shargabi, T., Andescavage, N. N., Metzler, M., Chang, T., et al. (2014). Heart rate variability in encephalopathic newborns during and after therapeutic hypothermia. J. Perinatol. 34, 836-841. doi: 10. 1038/jp.2014.108

McDuff, D., Gontarek, S., and Picard, R. W. (2014). Improvements in remote cardiopulmonary measurement using a five band digital camera. IEEE Trans. Biomed. Eng. 61, 2593-2601. doi: 10.1109/TBME.2014.2323695

McDuff, D. J., Blackford, E. B., and Estepp, J. R. (2018). Fusing partial camera signals for noncontact pulse rate variability measurement. IEEE Trans. Biomed. Eng. 65, 1725-1739. doi: 10.1109/TBME.2017.2771518

Metzler, M., Govindan, R., Al-Shargabi, T., Vezina, G., Andescavage, N., Wang, Y., et al. (2017). Pattern of brain injury and depressed heart rate variability in newborns with hypoxic ischemic encephalopathy. Pediatr. Res. 82, 438-443. doi: $10.1038 /$ pr.2017.94

Mizumoto, H., Tomotaki, S., Shibata, H., Ueda, K., Akashi, R., Uchio, H., et al. (2012). Electrocardiogram shows reliable heart rates much earlier than pulse oximetry during neonatal resuscitation. Pediatr. Int. 54, 205-207. doi: 10.1111/ j.1442-200X.2011.03506.x

Moorman, J. R., Carlo, W. A., Kattwinkel, J., Schelonka, R. L., Porcelli, P. J., Navarrete, C. T., et al. (2011). Mortality reduction by heart rate characteristic monitoring in very low birth weight neonates: a randomized trial. J. Pediatr. 159, 900.e1-906.e1. doi: 10.1016/j.jpeds.2011.06.044

Mowery, N. T., Morris, J. A. Jr., Jenkins, J. M., Ozdas, A., and Norris, P. R. (2011). Core temperature variation is associated with heart rate variability independent of cardiac index: a study of 278 trauma patients. J. Crit. Care 26, 534.e9-534.e17. doi: $10.1016 /$ j.jcrc.2010.11.008

Mulkey, S. B., and du Plessis, A. (2018). The critical role of the central autonomic nervous system in fetal-neonatal transition. Semin. Pediatr. Neurol. 28, 29-37. doi: 10.1016/j.spen.2018.05.004

Mulkey, S. B., and du Plessis, A. J. (2019). Autonomic nervous system development and its impact on neuropsychiatric outcome. Pediatr. Res. 85, 120-126. doi: 10.1038/s41390-018-0155-0

Mulkey, S. B., Govindan, R., Metzler, M., Swisher, C. B., Hitchings, L., Wang, Y., et al. (2020). Heart rate variability is depressed in the early transitional period for newborns with complex congenital heart disease. Clin. Auton. Res. 30, 165-172. doi: 10.1007/s10286-019-00616-w

Mulkey, S. B., Kota, S., Swisher, C. B., Hitchings, L., Metzler, M., Wang, Y., et al. (2018). Autonomic nervous system depression at term in neurologically normal premature infants. Early Hum. Dev. 123, 11-16. doi: 10.1016/j.earlhumdev. 2018.07.003
Munoz, M. L., van Roon, A., Riese, H., Thio, C., Oostenbroek, E., Westrik, I., et al. (2015). Validity of (Ultra-)short recordings for heart rate variability measurements. PLoS One 10:e0138921. doi: 10.1371/journal.pone.0138921

Nukaya, S., Sugie, M., Kurihara, Y., Hiroyasu, T., Watanabe, K., and Tanaka, H. (2014). A noninvasive heartbeat, respiration, and body movement monitoring system for neonates. Artif. Life Robot. 19, 414-419. doi: 10.1007/s10015-0140179-4

OECD (2020). Infant Mortality Rates (indicator). OECD: Paris, doi: 10.1787/ 83dea506-en

Okur, N., Uras, N., Buyuktiryaki, M., Oncel, M. Y., Saria, F. N., and Yarci, E. (2019). Neonatal pain and heart rate variability in preterm infants treated with surfactant: a pilot study. Arch. Argent. Pediatr. 117, 397-401. doi: 10.5546/aap. 2019.eng. 397

Oliveira, V., Martins, R., Liow, N., Teiserskas, J., von Rosenberg, W., Adjei, T., et al. (2019a). Prognostic accuracy of heart rate variability analysis in neonatal encephalopathy: a systematic review. Neonatology 115, 59-67. doi: 10.1159/ 000493002

Oliveira, V., von Rosenberg, W., Montaldo, P., Adjei, T., Mendoza, J., Shivamurthappa, V., et al. (2019b). Early postnatal heart rate variability in healthy newborn infants. Front. Physiol. 10:922. doi: 10.3389/fphys.2019.00922

Ostojic, D., Guglielmini, S., Moser, V., Fauchère, J. C., Bucher, H. U., Bassler, D., et al. (2020). "Reducing false alarm rates in neonatal intensive care: a new machine learning approach," in Oxygen Transport to Tissue XLI Advances in Experimental Medicine and Biology, eds P.-D. Ryu, J. C. LaManna, D. K. Harrison, and S.-S. Lee (Cham: Sringer International ublishing), 285-290. doi: 10.1007/978-3-030-34461-0_36

Otieno, P., Waiswa, P., Butrick, E., Namazzi, G., Achola, K., Santos, N., et al. (2018). Strengthening intrapartum and immediate newborn care to reduce morbidity and mortality of preterm infants born in health facilities in Migori County, Kenya and Busoga Region, Uganda: a study protocol for a randomized controlled trial. Trials 19:313. doi: 10.1186/s13063-018-2696-2

Ozawa, M., Sasaki, M., and Kanda, K. (2010). Effect of procedure light on the physiological responses of preterm infants. Jpn. J. Nurs. Sci. 7, 76-83. doi: 10.1111/j.1742-7924.2010.00142.x

Özdemir, R., Olukman, Ö, Karadeniz, C., Çelik, K., Katipoð̋lu, N., Muhtar Y $\iota$ lmazer, M., et al. (2018). Effect of unconjugated hyperbilirubinemia on neonatal autonomic functions: evaluation by heart rate variability. J. Matern. Fetal Neonatal Med. 31, 2763-2769. doi: 10.1080/14767058.2017.1355901

Pagani, M., Lucini, D., and Porta, A. (2012). Sympathovagal balance from heart rate variability: time for a second round? Exp. Physiol. 97, 1141-1142. doi: 10.1113/expphysiol.2012.066977

Passi, R., Doheny, K. K., Gordin, Y., Hinssen, H., and Palmer, C. (2017). Electrical grounding improves vagal tone in preterm infants. Neonatology 112, 187-192. doi: 10.1159/000475744

Patural, H., Pichot, V., Flori, S., Giraud, A., Franco, P., Pladys, P., et al. (2019). Autonomic maturation from birth to 2 years: normative values. Heliyon 5:e01300. doi: 10.1016/j.heliyon.2019.e01300

Paul, M., Karthik, S., Joseph, J., Sivaprakasam, M., Kumutha, J., Leonhardt, S., et al. (2020). Non-contact sensing of neonatal pulse rate using camera-based imaging: a clinical feasibility study. Physiol. Meas. 41:024001. doi: 10.1088/ 1361-6579/ab755c

Pernice, R., Javorka, M., Krohova, J., Czippelova, B., Turianikova, Z., Busacca, A., et al. (2018). Reliability of short-term heart rate variability indexes assessed through photoplethysmography. Conf. Proc. IEEE Eng. Med. Biol. Soc. 2018, 5513-5610. doi: 10.1109/EMBC.2018.8513634

Pernice, R., Javorka, M., Krohova, J., Czippelova, B., Turianikova, Z., Busacca, A., et al. (2019a). A validity and reliability study of conditional entropy measures of pulse rate variability. Conf. Proc. IEEE Eng. Med. Biol. Soc. 2019, 5568-5571. doi: 10.1109/EMBC.2019.8856594

Pernice, R., Javorka, M., Krohova, J., Czippelova, B., Turianikova, Z., Busacca, A., et al. (2019b). Comparison of short-term heart rate variability indexes evaluated through electrocardiographic and continuous blood pressure monitoring. Med. Biol. Eng. Comput. 57, 1247-1263. doi: 10.1007/s11517-019-0 1957-4

Phillipos, E., Solevåg, A. L., Pichler, G., Aziz, K., van Os, S., O’Reilly, M., et al. (2016). Heart rate assessment immediately after birth. Neonatology 109, 130138. doi: $10.1159 / 000441940$ 
Pichot, V., Roche, F., Celle, S., Barthélémy, J. C., and Chouchou, F. (2016). HRV analysis: a free software for analyzing cardiac autonomic activity. Front. Physiol. 7:557. doi: 10.3389/fphys.2016.00557

Pillai Riddell, R. R., Racine, N. M., Gennis, H. G., Turcotte, K., Uman, L. S., Horton, R. E., et al. (2015). Non-pharmacological management of infant and young child procedural pain. Cochrane Database Syst. Rev. 2015:CD006275. doi: 10.1002/14651858.CD006275.pub3

Pinaya, W. H. L., Mechelli, A., and Sato, J. R. (2019). Using deep autoencoders to identify abnormal brain structural patterns in neuropsychiatric disorders: a large-scale multi-sample study. Hum. Brain Mapp. 40, 944-954. doi: 10.1002/ hbm. 24423

Prietsch, V., Maier, R., Schmitz, L., and Obladen, M. (1992). Long-term variability of heart rate increases with successful closure of patent ductus arteriosus in preterm infants. Biol. Neonate 61, 142-149. doi: 10.1159/000243736

Raczyńska, A., and Gulczyńska, E. (2019). The impact of positioning on bottlefeeding in preterm infants $(=34 \mathrm{GA})$. A comparative study of the semi-elevated and the side-lying position - a pilot study. Dev. Period Med. 23, 117-124.

Rajalakshmi, A., Sunitha, K. A., and Venkataraman, R. (2019). A survey on neonatal incubator monitoring system. J. Phys. Conf. Ser. 1362:012128. doi: $10.1088 / 1742-6596 / 1362 / 1 / 012128$

Rashwan, N. I., Hassan, M. H., Mohey El-Deen, Z. M., and Ahmed, A. E. (2019). Validity of biomarkers in screening for neonatal sepsis - a single center -hospital based study. Pediatr. Neonatol. 60, 149-155. doi: 10.1016/j.pedneo.2018.05.001

Raynor, L. L., Saucerman, J. J., Akinola, M. O., Lake, D. E., Moorman, J. R., and Fairchild, K. D. (2012). Cytokine screening identifies NICU patients with Gram-negative bacteremia. Pediatr. Res. 71, 261-266. doi: 10.1038/pr.2011.45

Rees, C. A. (2014). Lost among the trees? The autonomic nervous system and paediatrics. Arch. Dis. Child. 99, 552-562. doi: 10.1136/archdischild-2012301863

Reynolds, R. M. (2013). Glucocorticoid excess and the developmental origins of disease: two decades of testing the hypothesis-2012 Curt Richter Award Winner. Psychoneuroendocrinology 38, 1-11. doi: 10.1016/j.psyneuen.2012.08. 012

Rivolta, M. W., Stampalija, T., Casati, D., Richardson, B. S., Ross, M. G., Frasch, M. G., et al. (2014). Acceleration and deceleration capacity of fetal heart rate in an in-vivo sheep model. PLoS One 9:e104193. doi: 10.1371/journal.pone. 0104193

Romano, M., Bifulco, P., Cesarelli, M., Sansone, M., and Bracale, M. (2006). Foetal heart rate power spectrum response to uterine contraction. Med. Biol. Eng. Comput. 44, 188-201. doi: 10.1007/s11517-006-0022-8

Sahni, R., Gupta, A., Ohira-Kist, K., and Rosen, T. S. (2003). Motion resistant pulse oximetry in neonates. Arch. Dis. Child Fetal Neonatal Ed. 88, F505-F508. doi: 10.1136/fn.88.6.f505

Schechtman, V. L., Raetz, S. L., Harper, R. K., Garfinkel, A., Wilson, A. J., Southall, D. P., et al. (1992). Dynamic analysis of cardiac R-R intervals in normal infants and in infants who subsequently succumbed to the sudden infant death syndrome. Pediatr. Res. 31, 606-612. doi: 10.1203/00006450-199206000-00014

Schmidt, A., Schukat-Talamazzini, E. G., Zöllkau, J., Pytlik, A., Leibl, S., Kumm, K., et al. (2018). Universal characteristics of evolution and development are inherent in fetal autonomic brain maturation. Auton. Neurosci. 212, 32-41. doi: 10.1016/j.autneu.2018.02.004

Schneider, U., Bode, F., Schmidt, A., Nowack, S., Rudolph, A., Dölker, E. M., et al. (2018). Developmental milestones of the autonomic nervous system revealed via longitudinal monitoring of fetal heart rate variability. PLoS One 13:e0200799. doi: 10.1371/journal.pone.0200799

Seely, A. J. E., Green, G. C., and Bravi, A. (2011). "Continuous multiorgan variability monitoring in critically ill patients \&\#x2014; complexity science at the bedside," in 2011 Annual International Conference of the IEEE Engineering in Medicine and Biology Society, Boston, MA: IEEE, 5503-5506. doi: 10.1109/ IEMBS.2011.6091404

Semenova, O., Carra, G., Lightbody, G., Boylan, G., Dempsey, E., and Temko, A. (2018). "Heart rate variability during periods of low blood pressure as a predictor of short-term outcome in preterms," in 2018 40th Annual International Conference of the IEEE Engineering in Medicine and Biology Society (EMBC), Honolulu, HI: IEEE, 5614-5517. doi: 10.1109/EMBC.2018. 8513600

Shaffer, F., and Ginsberg, J. P. (2017). An overview of heart rate variability metrics and norms. Front. Public Health 5:258. doi: 10.3389/fpubh.2017.00258
Shaffer, F., McCraty, R., and Zerr, C. L. (2014). A healthy heart is not a metronome: an integrative review of the heart's anatomy and heart rate variability. Front. Psychol. 5:1040. doi: 10.3389/fpsyg.2014.01040

Shin, J. H., Hwang, S. H., Chang, M. H., and Park, K. S. (2011). Heart rate variability analysis using a ballistocardiogram during Valsalva manoeuvre and post exercise. Physiol. Meas. 32, 1239-1264. doi: 10.1088/0967-3334/32/8/015

Shiroiwa, Y., Kamiya, Y., Uchibori, S., Inukai, K., Kito, H., Shibata, T., et al. (1986). Activity, cardiac and respiratory responses of blindfold preterm infants in a neonatal intensive care unit. Early Hum. Dev. 14, 259-265. doi: 10.1016/03783782(86) $90187-8$

Shuffrey, L. C., Myers, M. M., Odendaal, H. J., Elliott, A. J., du Plessis, C., Groenewald, C., et al. (2019). Fetal heart rate, heart rate variability, and heart rate/movement coupling in the safe passage study. J. Perinatol. 39, 608-618. doi: 10.1038/s41372-019-0342-9

Signorini, M. G., Fanelli, A., and Magenes, G. (2014). Monitoring fetal heart rate during pregnancy: contributions from advanced signal processing and wearable technology. Comput. Math. Methods Med. 2014, 1-10. doi: 10.1155/ 2014/707581

Simeoni, U., Armengaud, J. B., Siddeek, B., and Tolsa, J. F. (2018). Perinatal origins of adult disease. Neonatology 113, 393-399. doi: 10.1159/000487618

Singh, N., Moneghetti, K. J., Christle, J. W., Hadley, D., Plews, D., and Froelicher, V. (2018). Heart rate variability: an old metric with new meaning in the era of using mhealth technologies for health and exercise training guidance. part one: physiology and methods. Arrhythm. Electrophysiol. Rev. 7, 193-198. doi: 10.15420/aer.2018.27.2

Siren, P. M. A. (2017). SIDS-CDF hypothesis revisited: cause vs. Contributing factors. Front. Neurol. 7:244. doi: 10.3389/fneur.2016.00244

Spassov, L., Curzi-Dascalova, L., Clairambault, J., Kauffmann, F., Eiselt, M., Médigue, C., et al. (1994). Heart rate and heart rate variability during sleep in small-for-gestational age newborns. Pediatr. Res. 35, 500-505. doi: 10.1203/ 00006450-199404000-00022

Spilka, J., Chudáèe, V., Kouck $\imath$, M., Lhotská, L., Huptych, M., Jankù, P., et al. (2012). Using nonlinear features for fetal heart rate classification. Biomed. Signal Process. Control 7, 350-357. doi: 10.1016/j.bspc.2011.06.008

Spyridou, K., Chouvarda, I., Hadjileontiadis, L., and Maglaveras, N. (2018). Linear and nonlinear features of fetal heart rate on the assessment of fetal development in the course of pregnancy and the impact of fetal gender. Physiol. Meas. 39:015007. doi: 10.1088/1361-6579/aa9e3c

Stapelberg, N. J. C., Neumann, D. L., Shum, D. H. K., McConnell, H., and Hamilton-Craig, I. (2017). The sensitivity of 38 heart rate variability measures to the addition of artifact in human and artificial 24-hr cardiac recordings. Ann. Noninvasive Electrocardiol. 23:e12483. doi: 10.1111/anec.12483

Stenson, B. J. (2016). Oxygen saturation targets for extremely preterm infants after the NeOProM trials. Neonatology 109, 352-358. doi: 10.1159/000444913

Stéphan-Blanchard, E., Chardon, K., Léké, A., Delanaud, S., Bach, V., and Telliez, F. (2013). Heart rate variability in sleeping preterm neonates exposed to cool and warm thermal conditions. PLoS One 8:e68211. doi: 10.1371/journal.pone. 0068211

Stock, C., Teyssier, G., Pichot, V., Goffaux, P., Barthelemy, J. C., and Patural, H. (2010). Autonomic dysfunction with early respiratory syncytial virus-related infection. Auton. Neurosci. 156, 90-95. doi: 10.1016/j.autneu.2010.03.012

Stone, M. L., Tatum, P. M., Weitkamp, J. H., Mukherjee, A. B., Attridge, J., McGahren, E. D., et al. (2013). Abnormal heart rate characteristics before clinical diagnosis of necrotizing enterocolitis. J. Perinatol. 33, 847-850. doi: 10.1038/jp.2013.63

Sullivan, B. A., Grice, S. M., Lake, D. E., Moorman, J. R., and Fairchild, K. D. (2014). Infection and other clinical correlates of abnormal heart rate characteristics in preterm infants. J. Pediatr. 164, 775-780. doi: 10.1016/j.jpeds.2013.11.038

Sullivan, B. A., Wallman-Stokes, A., Isler, J., Sahni, R., Moorman, J. R., Fairchild, K. D., et al. (2018). Early pulse oximetry data improves prediction of death and adverse outcomes in a two-center cohort of very low birth weight infants. Am. J. Perinatol. 35, 1331-1338. doi: 10.1055/s-0038-1654712

Sun, Y., Hu, S., Azorin-Peris, V., Kalawsky, R., and Greenwald, S. (2012). Noncontact imaging photoplethysmography to effectively access pulse rate variability. J. Biomed. Opt. 18:061205. doi: 10.1117/1.JBO.18.6.061205

Sun, Y., and Thakor, N. (2016). Photoplethysmography revisited: from contact to noncontact, from point to imaging. IEEE Trans. Biomed. Eng. 63, 463-477. doi: 10.1109/TBME.2015.2476337 
Task Force of the European Society of Cardiology the North American Society of Pacing Electrophysiology (1996). Heart rate variability. standards of measurement, physiological interpretation, and clinical. (Use). Circulation 93, 1043-1065. doi: 10.1161/01.CIR.93.5.1043

Thayer, J. F., Ahs, F., Fredrikson, M., Sollers, J. J. III, and Wager, T. D. (2012). A meta-analysis of heart rate variability and neuroimaging studies: implications for heart rate variability as a marker of stress and health. Neurosci. Biobehav. Rev. 36, 747-756. doi: 10.1016/j.neubiorev.2011.11.009

Thayer, J. F., Hansen, A. L., Saus-Rose, E., and Johnsen, B. H. (2009). Heart rate variability, prefrontal neural function, and cognitive performance: the neurovisceral integration perspective on self-regulation, adaptation, and health. Ann. Behav. Med. 37, 141-153. doi: 10.1007/s12160-009-9101-z

Thayer, J. F., and Lane, R. D. (2000). A model of neurovisceral integration in emotion regulation and dysregulation. J. Affect. Disord. 61, 201-216. doi: 10. 1016/s0165-0327(00)00338-4

Thiriez, G., Mougey, C., Vermeylen, D., Wermenbol, V., Lanquart, J. P., Lin, J. S., et al. (2015). Altered autonomic control in preterm newborns with impaired neurological outcomes. Clin. Auton. Res. 25, 233-242. doi: 10.1007/s10286-0150298-6

Thoyre, S. M., Holditch-Davis, D., Schwartz, T. A., Melendez Roman, C. R., and Nix, W. (2012). Coregulated approach to feeding preterm infants with lung disease: effects during feeding. Nurs. Res. 61, 242-251. doi: 10.1097/NNR. 0b013e31824b02ad

Topalidou, A., Ali, N., Sekulic, S., and Downe, S. (2019). Thermal imaging applications in neonatal care: a scoping review. BMC Pregnancy Childbirth. 19:381. doi: 10.1186/s12884-019-2533-y

Tribe, R. M., Taylor, P. D., Kelly, N. M., Rees, D., Sandall, J., and Kennedy, H. P. (2018). Parturition and the perinatal period: can mode of delivery impact on the future health of the neonate? J. Physiol. 596, 5709-5722. doi: 10.1113/JP2 75429

Tu, W., Chen, P. A., Koenig, N., Gomez, D., Fujiwara, E., Gill, M. J., et al. (2020). Machine learning models reveal neurocognitive impairment type and prevalence are associated with distinct variables in HIV/AIDS. J. Neurovirol. 26, 41-51. doi: 10.1007/s13365-019-00791-6

Turova, V., Sidorenko, I., Eckardt, L., Rieger-Fackeldey, E., Felderhoff-Müser, U., Alves-Pinto, A., et al. (2020). Machine learning models for identifying preterm infants at risk of cerebral hemorrhage. PLoS One 15:e0227419. doi: 10.1371/ journal.pone.0227419

Uhrikova, Z., Zibolen, M., Javorka, K., Chladekova, L., and Javorka, M. (2015). Hyperbilirubinemia and phototherapy in newborns: effects on cardiac autonomic control. Early Hum. Dev. 91, 351-356. doi: 10.1016/j.earlhumdev. 2015.03.009

United Nations Inter-agency Group for Child Mortality Estimation (UN IGME) (2019). ). Levels \& Trends in Child Mortality: Report 2019, Estimates developed by the United Nations Inter-agency Group for Child Mortality Estimation. New York, NY: United Nations Children's Fund.

Urfer-Maurer, N., Ludyga, S., Stalder, T., Brand, S., Holsboer-Trachsler, E., Gerber, M., et al. (2018). Heart rate variability and salivary cortisol in very preterm children during school age. Psychoneuroendocrinology 87, 27-34. doi: 10.1016/ j.psyneuen.2017.10.004

Valenza, G., Iozzia, L., Cerina, L., Mainardi, L., and Barbieri, R. (2018). Analysis of instantaneous linear, nonlinear and complex cardiovascular dynamics from videophotoplethysmography. Methods Inf. Med. 57, 135-140. doi: 10.3414/ ME17-02-0013

van Laar, J., Peters, C., Vullings, R., Houterman, S., Bergmans, J., and Oei, S. (2010). Fetal autonomic response to severe acidaemia during labour: fetal autonomic stress response during labour. BJOG Int. J. Obstet. Gynaecol. 117, 429-437. doi: 10.1111/j.1471-0528.2009.02456.x

van Laar, J. O. E. H., Peters, C. H. L., Houterman, S., Wijn, P. F. F., Kwee, A., and Oei, S. G. (2011). Normalized spectral power of fetal heart rate variability is associated with fetal scalp blood pH. Early Hum. Dev. 87, 259-263. doi: 10.1016/j.earlhumdev.2011.01.028

Van Leeuwen, P., Werner, L., Hilal, Z., Schiermeier, S., Hatzmann, W., and Grönemeyer, D. (2014). Fetal electrocardiographic measurements in the assessment of fetal heart rate variability in the antepartum period. Physiol. Meas. 35, 441-454. doi: 10.1088/0967-3334/35/3/441

Van Puyvelde, M., Collette, L., Gorissen, A. S., Pattyn, N., and McGlone, F. (2019a). Infants autonomic cardio-respiratory responses to nurturing stroking touch delivered by the mother or the father. Front. Physiol. 10:1117. doi: 10.3389/ fphys.2019.01117

Van Puyvelde, M., Gorissen, A. S., Pattyn, N., and McGlone, F. (2019b). Does touch matter? The impact of stroking versus non-stroking maternal touch on cardio-respiratory processes in mothers and infants. Physiol. Behav. 207, 55-63. doi: 10.1016/j.physbeh.2019.04.024

van Ravenswaaij-Arts, C. M., Hopman, J. C., Kollée, L. A., van Amen, J. P., and Stoelinga, G. B. (1991). The influence of respiratory distress syndrome on heart rate variability in very preterm infants. Early Hum. Dev. 27, 207-221. doi: 10.1016/0378-3782(91)90195-9

Vassar, R., Schadl, K., Cahill-Rowley, K., Yeom, K., Stevenson, D., and Rose, J. (2020). Neonatal brain microstructure and machine-learning-based prediction of early language development in children born very preterm. Pediatr. Neurol. 108, 86-92. doi: 10.1016/j.pediatrneurol.2020.02.007

Venkataraman, R., Kamaluddeen, M., Amin, H., and Lodha, A. (2018). Is Less noise, light and parental/caregiver stress in the neonatal intensive care unit better for neonates? Indian Pediatr. 55, 17-21. doi: 10.1007/s13312-018-1220-9

Verklan, M. T., and Walden, M. (2014). Core Curriculum for Neonatal Intensive Care Nursing, 5th Edn. St. Louis, MO: Elsevier.

Villarroel, M., Guazzi, A., Jorge, J., Davis, S., Watkinson, P., Green, G., et al. (2014). Continuous non-contact vital sign monitoring in neonatal intensive care unit. Healthc. Technol. Lett. 1, 87-91. doi: 10.1049/htl.2014.0077

Voss, A., Schroeder, R., Vallverdú, M., Schulz, S., Cygankiewicz, I., Vázquez, R., et al. (2013). Short-term vs. long-term heart rate variability in ischemic cardiomyopathy risk stratification. Front. Physiol. 4:364. doi: 10.3389/fphys. 2013.00364

Walusinski, O. (2006). Yawning: unsuspected avenue for a better understanding of arousal and interoception. Med. Hypotheses 67, 6-14. doi: 10.1016/j.mehy.2006. 01.020

Wang, K., Zhu, T., Zhang, X., Yu, C., Cao, X., Tang, J., et al. (2015). [Comparison of heart rate variability measurements between ballistocardiogram and electrocardiography]. Zhonghua Xin Xue Guan Bing Za Zhi 43, 448-451.

Wang, Y., Carrault, G., Beuchee, A., Costet, N., Shu, H., and Senhadji, L. (2016). Heart rate variability and respiration signal as diagnostic tools for late onset sepsis in neonatal intensive care units. ArXiv [Preprint] Available online at: https://arxiv.org/abs/1605.05247 (accessed July 25, 2020).

Warmerdam, G. J. J., Vullings, R., Van Laar, J. O. E. H., Van der Hout-Van der Jagt, M. B., Bergmans, J. W. M., Schmitt, L., et al. (2016). Using uterine activity to improve fetal heart rate variability analysis for detection of asphyxia during labor. Physiol. Meas. 37, 387-400. doi: 10.1088/0967-3334/37/3/387

Warmerdam, G. J. J., Vullings, R., Van Laar, J. O. E. H., Van der Hout-Van der Jagt, M. B., Bergmans, J. W. M., Schmitt, L., et al. (2018). Detection rate of fetal distress using contraction-dependent fetal heart rate variability analysis. Physiol. Meas. 39:025008. doi: 10.1088/1361-6579/aaa925

Watson, G., Patel, K., Leng, D., Vanna, D., Khut, S., Prak, M., et al. (2020). Barriers and facilitators to neonatal health and care-seeking behaviours in rural Cambodia: a qualitative study. BMJ Open 10:e035449. doi: 10.1136/bmjopen2019-035449

Weber, A., and Harrison, T. M. (2019). Reducing toxic stress in the neonatal intensive care unit to improve infant outcomes. Nurs. Outlook 67, 169-189. doi: 10.1016/j.outlook.2018.11.002

Weber, F., Roeleveld, H. G., Geerts, N. J. E., Warmenhoven, A. T., Schröder, R., and de Leeuw, T. G. (2019). The heart rate variability-derived Newborn Infant Parasympathetic Evaluation (NIPE ${ }^{\mathrm{TM}}$ ) Index in pediatric surgical patients from 0 to 2 years under sevoflurane anesthesia-A prospective observational pilot study. Paediatr. Anaesth. 29, 377-384. doi: 10.1111/pan.13613

Weese-Mayer, D. E., Corwin, M. J., Peucker, M. R., Di Fiore, J. M., Hufford, D. R., Tinsley, L. R., et al. (2000). Comparison of apnea identified by respiratory inductance plethysmography with that detected by end-tidal $\mathrm{CO}(2)$ or thermistor. The CHIME Study Group. Am. J. Respir. Crit. Care Med. 162(2 Pt 1), 471-480. doi: 10.1164/ajrccm.162.2.9904029

Weissman, A., Zimmer, E. Z., Aranovitch, M., and Blazer, S. (2012). Heart rate dynamics during acute pain in newborns. Pflugers. Arch. 464, 593-599. doi: 10.1007/s00424-012-1168-x

Werth, J. V. S. W. (2019). On the Automated Analysis of Preterm Infant Sleep States From Electrocardiography. Eindhoven: Technische Universiteit Eindhoven.

Werther, T., Aichhorn, L., Baumgartner, S., Berger, A., Klebermass-Schrehof, K., and Salzer-Muhar, U. (2018). Discrepancy between invasive and non-invasive 
blood pressure readings in extremely preterm infants in the first four weeks of life. PLoS One 13:e0209831. doi: 10.1371/journal.pone.0209831

WHO (2016). World Health Statistics 2016. Monitoring Health for The SDGs. Avaliable online at: http://apps.who.int/iris/bitstream/10665/206498/1/9789 241565264_eng.pdf (accessed July 28, 2020).

WHO (2017). Reaching the Every Newborn National 2020 Milestones: Country Progress, Plans and Moving Forward. Avaliable online at: https://apps.who.int/ iris/bitstream/handle/10665/255719/9789241512619-eng.pdf (accessed July 28, 2020).

Williams, A. L., Sanderson, M., Lai, D., Selwyn, B. J., and Lasky, R. E. (2009). Intensive care noise and mean arterial blood pressure in extremely lowbirth-weight neonates. Am. J. Perinatol. 26, 323-329. doi: 10.1055/s-0028-110 4741

Williams, D. P., Koenig, J., Carnevali, L., Sgoifo, A., Jarczok, M. N., Sternberg, E. M., et al. (2019). Heart rate variability and inflammation: a meta-analysis of human studies. Brain Behav. Immun. 80, 219-226. doi: 10.1016/j.bbi.2019.03.009

Xu, A., Durosier, L. D., Ross, M. G., Hammond, R., Richardson, B. S., and Frasch, M. G. (2014). Adaptive brain shut-down counteracts neuroinflammation in the near-term ovine fetus. Front. Neurol. 5:110. doi: 10.3389/fneur.2014.00110

Yamaguchi, K., Lear, C. A., Beacom, M. J., Ikeda, T., Gunn, A. J., and Bennet, L. (2018). Evolving changes in fetal heart rate variability and brain injury after hypoxia-ischaemia in preterm fetal sheep. J. Physiol. 596, 6093-6104. doi: 10. 1113/JP275434

Yiallourou, S. R., Sands, S. A., Walker, A. M., and Horne, R. S. (2012). Maturation of heart rate and blood pressure variability during sleep in term-born infants. Sleep 35, 177-186. doi: 10.5665/sleep.1616

Zahr, L. K., and de Traversay, J. (1995). Premature infant responses to noise reduction by earmuffs: effects on behavioral and physiologic measures. J. Perinatol. 15, 448-455.
Zhang, K., Wang, S., Wu, L., Song, Y., Cai, M., Zhang, M., et al. (2019). Newborn infant parasympathetic evaluation (NIPE) as a predictor of hemodynamic response in children younger than 2 years under general anesthesia: an observational pilot study. BMC Anesthesiol. 19:98. doi: 10.1186/s12871-0190774-y

Zhao, F., Li, M., Jiang, Z., Tsien, J. Z., and Lu, Z. (2016). Camera-based, noncontact, vital-signs monitoring technology may provide a way for the early prevention of SIDS in infants. Front. Neurol. 7:236. doi: 10.3389/fneur.2016. 00236

Zöllkau, J., Dölker, E.-M., Schmidt, A., Schneider, U., and Hoyer, D. (2019). Dependencies between maternal and fetal autonomic tone. J. Perinat. Med. 47, 323-330. doi: 10.1515/jpm-2018-0221

Zuzarte, I., Indic, P., Sternad, D., and Paydarfar, D. (2019). Quantifying movement in preterm infants using photoplethysmography. Ann. Biomed. Eng. 47, 646658. doi: 10.1007/s10439-018-02135

Conflict of Interest: DB was employed by the company BioTekna.

The remaining authors declare that the research was conducted in the absence of any commercial or financial relationships that could be construed as a potential conflict of interest.

Copyright (c) 2020 Chiera, Cerritelli, Casini, Barsotti, Boschiero, Cavigioli, Corti and Manzotti. This is an open-access article distributed under the terms of the Creative Commons Attribution License (CC BY). The use, distribution or reproduction in other forums is permitted, provided the original author(s) and the copyright owner(s) are credited and that the original publication in this journal is cited, in accordance with accepted academic practice. No use, distribution or reproduction is permitted which does not comply with these terms. 